\title{
Supercritical fluid extraction of bioactive compounds
}

\author{
Rui P.F.F. da Silva ${ }^{a^{*}}$, Teresa A.P. Rocha-Santos ${ }^{a, b}$, Armando C. Duarte $^{a, b}$ \\ ${ }^{a}$ Department of Chemistry, University of Aveiro, 3810-193 Aveiro, Portugal \\ ${ }^{\mathrm{b}}$ CESAM - Center for Environmental and Marine Studies, University of Aveiro, 3810-193 Aveiro, Portugal \\ ${ }^{*}$ Corresponding author: Rui P.F.F. da Silva \\ E-mail address: rpffds@gmail.com
}

\begin{abstract}
An ideal extraction method should be swift, yield quantitative recovery without degradation, and the extracts should be easily separated from the solvent. The development and application of alternative green technology to replace conventional extraction methods with improved extraction efficiency and low environmental impact for the determination of natural bioactive compounds is therefore, highly important. Supercritical fluid technology offers features that overcome many limitations of conventional extraction methods.

This review presents an analytical overview regarding the application of supercritical fluids in the extraction of bioactive compounds and their operative extraction conditions, along with the investigation of further improvements on the extraction efficiency and the applied techniques for the structural characterization and identification of such bioactive compounds.
\end{abstract}




\section{Keywords:}

Supercritical Fluid Extraction, Green Extraction, Extraction, Bioactive Compounds, Optimization, Experimental Design, Structural characterization.

\section{Introduction}

The quest for bioactive compounds from natural sources has been driven by scientific research of these targeted molecules against a vast array of diseases besides their use in food science and technology. The increasing evidence correlating diet and chronic illnesses, the limitation imposed on the application of food additives produced from synthetic chemicals as well as their banishment in some food products, made incorporation of natural food additives as one of the most important trends in the food industry today [1-4]. Many compounds extracted from natural sources have been shown to possess several bioactive applications, such as antimicrobial, antibacterial, antifungal, antiviral, anti-inflammatory, antitumor, antiobesity, anticholinesterase, phagocytotic, insecticide, and antioxidant functions.

One of the most studied bioactivities is the anti-proliferative activity of polyphenols. Phenolic compounds extracted from Rosmarinus officinalis is one case study that revealed antitumor activity, mainly due to the presence of several phenolic compounds, such as rosmarinic and carnosic acids, carnosol, rosmanol, epirosmanol and methyl carnosate among others [5]. Pharmacological studies performed with Codia verbenacea have revealed anti-inflammatory activity, and this effect was credited to the presence of $\alpha$ caryophyllene and trans-caryophyllene [6]. Another example is the antiviral properties obtained from thyme species (Thymus vulgaris, Thymus hyemalis and Thymus zygis) that have been associated with a high percentage of thymol, carvacrol and borneol present in the extracts [7]. Salvia officinalis represents another case study that have shown anti-inflammatory capabilities that were linked to camphor, borneol and 1,8-cineol [8]. Perillyl alcohol, a hydroxylated monocyclic monoterpene, found in Prunus avium, has been shown to be effective against the formation and progression of a variety of cancers [9]. Table 1 presents a compilation of different chemical class structures of selected bioactive compounds extracted from different sources that embody distinct bioactive applications.

The medicinal and pharmacological actions are often dependent on the presence of bioactive compounds called secondary metabolites [10-12]. Unlike the universal macromolecules of primary metabolism (e.g. monosaccharaides, polysaccharides, amino acids, proteins and lipids), which are commonly present in all organisms, secondary metabolites have a far more limited distribution in nature, are not necessarily produced under all conditions, and can only be found in specific organisms or group of organisms. The organism can produce these compounds either to protect itself within its own living ecosystem or they can play a basic role on its everyday existence while revealing bioactivity in unrelated biological systems. Although these compounds may have some vital role on the welfare of the producer, in the vast majority of cases their benefit to the organism is not yet known [13-16].

Secondary metabolites with reported medicinal properties consist of waxes, fatty acids, alkaloids, terpenoids, phenolics (simple phenolics and flavonoids), glycosides and phytosterols $[10,16]$.

Table 1 - Selected examples of bioactive structures of natural products extracted from several sources.

\begin{tabular}{|c|c|c|c|c|}
\hline Compound & Organism & $\begin{array}{c}\text { Class/structural } \\
\text { biogenic type }\end{array}$ & Seference \\
\hline
\end{tabular}




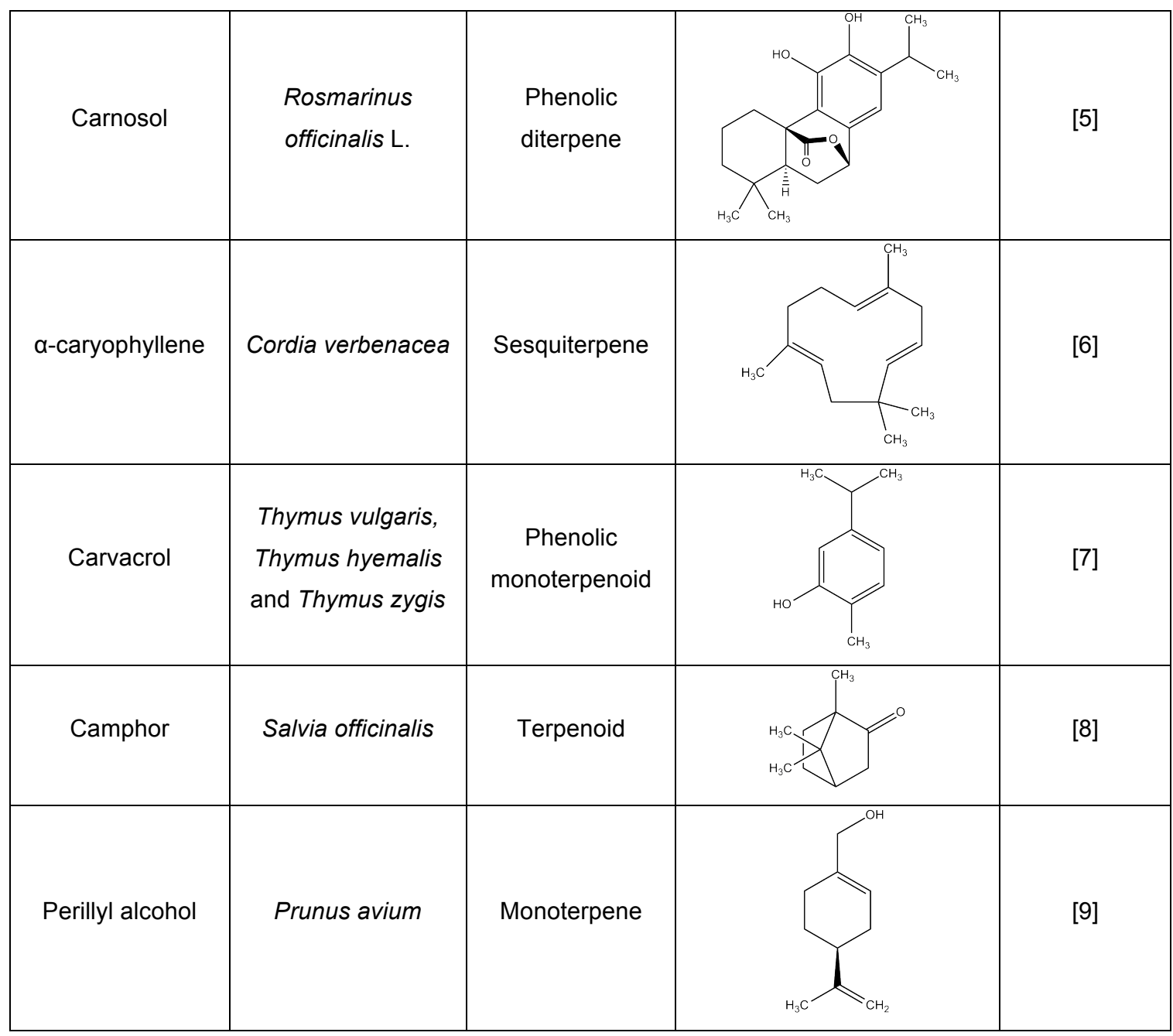

In order to obtain compounds with bioactive interest, the extraction and recovery of a solute from a solid matrix can be accomplished by a three-stage process: (1) local desorption of the solutes and their solubilization; (2) internal solute diffusion through the solid phase until its surface; and (3) external solute diffusion through the film. To achieve an efficient and adequate strategy of extraction, careful control of analytical conditions and optimization of each step is required, specifically, the sample collection should be carefully controlled as it is often neglected when compared to the handling of samples in the laboratory [17]. Moreover in matrices such as plant materials the extraction rate may be limited by either the solubilization or the diffusional step. Therefore, the optimization approach will strongly depend on the nature of the studied matrix $[4,17,18]$. Additionally, and despite the long practice history of herbs and other natural medicine, the utilization of a whole plant or any other crude preparation for experiments can possess several drawbacks. A simple variation on the collected biomass geographical area, harvest season, used/studied morphological part as well as climate and ecological system, can alter the constitution of the extract and therefore its bioactive capabilities. Furthermore, bioactivity changes or degradation due to inconsistency in collection, storage, and preparation of the raw material can also represent a weakness in the process of isolating biologically active products [15].

Apart from the matrices nature, the quality of the extract depends also on several other factors. The type, time and temperature of extraction, as well as the solvent nature and the solvent-to-sample ratio will affect the quantity and the composition of the extract. A good solvent includes low toxicity, low boiling point, rapid mass transfer of the solutes, conservative action and inability to cause the extract to complex or dissociate. 
In order to choose a solvent wisely, one must take into consideration the quantity of compounds to be extracted, the rate of the extraction, the diversity of extracted compounds, the ease of handling, the level of toxicity to the bioassay process, and the its health hazardous potential [19].

Conventional extraction methods such as Soxhlet and maceration using hazardous liquid solvents such as dichloromethane and methanol are time consuming and usually require several hours or even days to achieve a complete extraction[20]. Pedersen et al. [21] investigated the Soxhlet extraction of acrylamide from potato chips which took 7 days to achieve a constant concentration of the targeted compound. Conventional extractions also result in dilute extracts, thus demanding concentration of the extracted solute in the liquid solvent, and they may not result in quantitative recovery of the target solutes. Moreover, an ideal extraction method should be swift, yield quantitative recovery of target solute without degradation, and the solvent should be easily separated from the solute. Therefore, the development of alternative green analytical techniques has become a hot research topic in order to replace conventional extraction methods [3].

Green extraction has been focused mainly on the discovery and design of new extraction processes with reduced energy requirements, resorting to non-hazardous alternative solvents and renewable natural products while ensuring a safe and high quality extracts [22]. Supercritical fluid extraction (SFE) provides attractive features overcoming many of the limitations of conventional extractions $[18,20]$. Several reviews have been published on SFE fundamentals, experimental design and specific applications on food processing, surface coating analysis, vegetable matrices, extraction of metals as complexes, functional ingredients from natural sources, constituents of fish oil and decontamination of hazardous substances $[3,23-41]$. Therefore, taking the present framework into consideration there is still scope and interest in the publication of a review article covering the supercritical fluid extraction of natural compounds from both terrestrial and marine origin having several types of bioactivity, such as antioxidant, antitumor, antibacterial, antiviral, antimicrobial, anti-inflammatory and anticholinesterase. This work reviews, from an analytical standpoint in the period from 2010 to 2015, the basic principles, advantages, and technological advancements in the supercritical fluid extraction of bioactive compounds from natural sources rather than a purely process-related standpoint. Also, this review focuses specifically on works where supercritical fluid extraction raises bioactive extracts or compounds that have been successfully chemical characterized.

\section{Supercritical fluid extraction (SFE) for the discovery of bioactive compounds}

SFE provides several operational advantages over conventional extraction methods since it uses supercritical solvents, with different physicochemical properties such as density, diffusivity, viscosity and dielectric constant. Due to their low viscosity and relatively high diffusivity, supercritical fluids have enhanced transport properties than liquids, can diffuse easily through solid materials and can therefore give faster extraction rates. One of the main characteristics of a supercritical fluid is the possibility of modifying the density of the fluid by changing its pressure and/or temperature. Since density is related to solubility, by altering the extraction pressure, the solvent strength of the fluid can be modified. Other advantages, compared to other extraction techniques, are the use of solvents generally recognized as safe (GRAS), the higher efficiency of the extraction process in terms of increasing yields and lower extraction times, and the possibility of direct coupling with analytical chromatographic techniques such as gas chromatography (GC) or supercritical fluid chromatography (SFC) [26]. 
As listed in Table 2 there are several compounds that can be used as supercritical fluids, though carbon dioxide is the most commonly used solvent for three major reasons: firstly, it is innocuous to human health and to environment, respecting the sustainability criteria that increasingly governs the suitability of chemical processes; secondly, its moderate critical temperature $\left(31.2^{\circ} \mathrm{C}\right)$ is a key issue for the preservation of bioactive compounds in extracts; finally, the extract is preserved from contact with air, where light reactions of oxidation may occur [42].

Table 2 - Critical properties of some solvents used in SFE.[26]

\begin{tabular}{|c|c|c|c|c|}
\hline \multirow[b]{2}{*}{ Solvent } & \multicolumn{4}{|c|}{ Critical Property } \\
\hline & $\begin{array}{c}\text { Temperature } \\
\left({ }^{\circ} \mathrm{C}\right)\end{array}$ & $\begin{array}{c}\text { Pressure } \\
\text { (atm) }\end{array}$ & $\begin{array}{c}\text { Density } \rho_{\mathrm{SCF}} \\
(\mathrm{g} / \mathrm{mL})\end{array}$ & $\begin{array}{c}\text { Solubility } \sigma_{\mathrm{SFC}} \\
\left(\mathrm{cal}^{-1 / 2} \mathrm{~cm}^{-3 / 2}\right)\end{array}$ \\
\hline Carbon Dioxide & 31.2 & 72.9 & 0.470 & 7.5 \\
\hline Ethane & 32.4 & 48.2 & 0.200 & 5.8 \\
\hline Ethene & 10.1 & 50.5 & 0.200 & 5.8 \\
\hline Methanol & -34.4 & 79.9 & 0.272 & 8.9 \\
\hline Nitrous Oxide & 36.7 & 71.7 & 0.460 & 7.2 \\
\hline n-Butene & -139.9 & 36.0 & 0.221 & 5.2 \\
\hline n-Pentane & -76.5 & 33.3 & 0.237 & 5.1 \\
\hline Sulfur hexafluoride & 45.8 & 37.7 & 0.730 & 5.5 \\
\hline Water & 101.1 & 217.6 & 0.322 & 13.5 \\
\hline
\end{tabular}

Since carbon dioxide is a gas at room temperature, when the extraction is completed and the system decompressed, the elimination of $\mathrm{CO}_{2}$ is achieved, yielding a solvent-free extract. On an industrial scale, when carbon dioxide consumption is high, the operation can be controlled to recycle it. However, because of its low polarity, $\mathrm{CO}_{2}$ is less effective in extracting more polar compounds from natural matrices, and modifiers (also called cosolvents) are commonly used in order to overcome this problem [27,43]. These are polar compounds that, added in small amounts, can induce substantial changes of the solvent properties of pure supercritical $\mathrm{CO}_{2}$ [26,42]. Supercritical extraction basically occurs in two steps: the solubilization of the chemical compounds present on the solid matrix and its separation into the supercritical solvent. During the extraction, the solvent flows through the packed bed, solubilizing the existing compounds present in the matrix. Afterwards the solvent exits the extractor carrying the solubilized compounds, and by pressure reduction and/or temperature increase, the extract becomes solvent free.

According to Brunner [44] the solubilization process of a vegetal matrix occurs in various stages. Firstly, the vegetal matrix absorbs the supercritical solvent, swelling its cellular structure, membranes and dilating the intercellular channels, leading to a drop in the resistance to mass transfer; in the meantime, dissolution of the extract occurs, and mass transfer takes place from the inner matrix to its surface and the solubilized compounds reach the external surface. These are then transported from the surface to the supercritical solvent and finally removed from the solvent.

The optimization of SFE requires fundamental knowledge about thermodynamic data (solubility and selectivity) along with kinetic data (mass transfer coefficients). The kinetic representation of a SFE is obtained from the extraction curve data, which is normally represented in a graph of accumulated extracted mass versus time of extraction. The obtained curve depends on the process parameters and the phenomena that take place in the fixed bed reactor during the extraction process.

The extraction curve trend can easily be affected by solvent flow rate and bed particles size making it difficult to compare curves obtained from different raw materials and different instrumental setups. 
However, diverse information supplied by extraction curves, such as the constant extraction rate duration, allows the comparison between different experiments with the same substrate and equipment [44].

The study of supercritical extraction curves and the knowledge of the effects of the operational variables allow the establishment of the extractor volume and solvent flow rate (QCO2). According to several researchers [45-48], the overall extraction curves (OEC), are clearly divided into three periods (as shown in Figure 1) controlled by different mass transfer mechanisms:

a) Constant Extraction Rate (CER) period, where the external surface of the particles is covered with solute (easily accessible solute) and the convection is the dominant mechanism of mass transfer;

b) Falling Extraction Rate (FER) period, where failures in the external surface oil layer appear and the diffusion mechanism starts, operating combined with convection;

c) Low Extraction Rate (LER) or Diffusion-Controlled (DC) period, where the external layer of oil practically disappeared and the mass transfer occurs mainly by diffusion inside the solid particles

Porto et al.[49] in an attempt to evaluate the effect of temperature under SFE $\left(\mathrm{CO}_{2}\right)$ conditions such as 10 $\mathrm{MPa}$ and $15 \%$ water as cosolvent obtained the overall extraction curves (phenols vs. time) and observed that all curves exhibit the three periods (CER, FER and DC). The authors verified that: (a) up to 120 min the extraction curves start to diverge, (b) from 120 to $140 \mathrm{~min}$ a transition period where both mass transfer and phase equilibrium control de extractions; (c) up to $240 \mathrm{~min}$ a third smooth asymptotic period due to diffusion phenomena where the slopes depend on particle size and solvent flow rate. The phenols diffusion to the particle surface was slow since it has been verified that $10 \%$ of the final extracted phenols were deposited inside the grape marc particles. The phenols concentration decreased from 628.1 to $576.3 \mathrm{mg}$ $\mathrm{GAE} / 100 \mathrm{~g}$ DM with the increase of temperature from 313.15 to $333.15 \mathrm{~K}$, since an increase in temperature reduce the density of the modified supercritical $\mathrm{CO}_{2}\left(\mathrm{scCO}_{2}\right)$ and its solvent power. The authors also established the OEC at temperature of $313.15 \mathrm{~K}$ and pressure of 10 and $20 \mathrm{MPa}$ in order to evaluate the effect of temperature and observed that the extraction of phenols was higher at $10 \mathrm{MPa}$ $(403,5 \mathrm{mg} \mathrm{GAE} / 100 \mathrm{~g} \mathrm{DM})$ than at $20 \mathrm{MPa}$ (272.4 mg GAE/100 $\mathrm{g} \mathrm{DM})$. These results can be partially attributed to the low dispersion coefficient of the modified $\mathrm{scCO}_{2}$ which accounts for the axial and radial diffusion mechanisms and to the high porosity of the extraction bed thus reducing the contact between the solvent and the compounds to be extracted causing a loss of process efficiency. From these extraction curves the authors were able to choose the best temperature and pressure operating conditions to extract grape marc phenols.

Bioactive compounds which can also be referred as nutraceuticals, due to their existence in the human diet and their biological activity, are essential and non-essential compounds that are produced in nature and can be shown to have a therapeutic and toxic activity on human and animal diseases [18,50]. These bioactive natural products can be secondary metabolites produced by the organism in order to protect itself from adverse environmental conditions, or they can be compounds fundamental to its everyday existence but with unforeseen activity in unrelated biological systems [15]. The astonishing versatility of supercritical fluid technology renders it an excellent tool to obtain substances of therapeutic nature, allowing the isolation and processing of drugs into new medicines for specific drug delivery. Supercritical fluid extracts has also provided positive and promising results in applications in the food, cosmetics and pharmaceutical industries with the production of flavor enhancers, analgesics and anti-inflammatory drugs as well as the search for treatments for drastic diseases such as stroke, cancer and Alzheimer [18,39].

Table 3 shows a selection of different bioactive compounds extracted by SFE methodology from distinct sample sources where the main bioactivities are antioxidant $(41 \%)$, antitumor $(18 \%)$ and antibacterial activity $(10 \%)$, followed by antiviral, antimicrobial, anti-inflammatory and anticholinesterase (total of $5 \%$ ). 
Table 3 - Selected examples of bioactive compounds extracted from several sources using supercritical fluid extraction.

\begin{tabular}{|c|c|c|c|c|c|c|c|c|c|c|}
\hline \multirow{2}{*}{$\begin{array}{l}\text { Sample } \\
\text { Source }\end{array}$} & \multicolumn{6}{|c|}{ SFE conditions } & \multirow[b]{2}{*}{ Bioactivity } & \multirow[b]{2}{*}{ Target/Main Compounds } & \multirow[b]{2}{*}{ SFE results } & \multirow{2}{*}{ Ref. } \\
\hline & Solvent & $\begin{array}{c}\text { Temp } \\
\left({ }^{\circ} \mathrm{C}\right)\end{array}$ & $\mathrm{P}$ (bar) & $\begin{array}{l}\text { Flow } \\
\text { Rate }\end{array}$ & $\begin{array}{l}\text { Time } \\
(\min )\end{array}$ & Cosolvent & & & & \\
\hline Bidens pilosa & $\mathrm{CO}_{2}$ & 40 & 250 & $15 \mathrm{~g} / \mathrm{min}$ & 240 & - & $\begin{array}{l}\text { Antitumor (MCF-7 breast } \\
\text { cancer cells) }\end{array}$ & Polyacetylenes & $\eta_{\text {total }}=2.00 \%$ & {$[51]$} \\
\hline $\begin{array}{l}\text { Apium } \\
\text { graveolens }\end{array}$ & $\mathrm{CO}_{2}$ & 40 & 100 & $0.30 \mathrm{~kg} / \mathrm{h}$ & - & - & $\begin{array}{l}\text { Antimicrobial (S. aureus, } \\
\text { L. monocytogenes and } \\
\text { Listeria ivanovii strains) }\end{array}$ & $\begin{array}{l}\text { sedanenolide, sedanolide, } \\
\text { 3- } n \text {-butylphthalide }\end{array}$ & $\eta_{\text {total }}=2.74 \%$ & [1] \\
\hline Ceratonia siliqua & $\mathrm{CO}_{2}$ & 40 & 220 & $0.29 \mathrm{~kg} / \mathrm{h}$ & 450 & $\begin{array}{c}10 \% \\
(\text { Ethanol/water } \\
(8: 2))\end{array}$ & $\begin{array}{c}\text { Antitumor } \\
\text { (rat N1E-115 } \\
\text { neuroblastoma cells, } \\
\text { human HeLa cervical } \\
\text { cancer cell, MCF-7 breast } \\
\text { cancer cells) } \\
\end{array}$ & $\begin{array}{l}\text { Cinnamic acid, chrysoeriol, } \\
\text { tricetin-3',5'-dimethyl ether, } \\
\text { naringenin and ferulic acid }\end{array}$ & $\begin{array}{c}\eta_{\text {total }}=0.33 \% \\
\mathrm{AA}=0.130 \mathrm{mmol} \mathrm{TEAC} / \mathrm{g}_{\text {dry mas }}\end{array}$ & {$[52]$} \\
\hline Citrus sinensis & $\mathrm{CO}_{2}$ & 40 & 200 & $17 \mathrm{~g} / \mathrm{min}$ & 300 & - & $\begin{array}{c}\text { Antimicrobial } \\
\text { (Staphylococcus aureus) }\end{array}$ & $\begin{array}{l}\text { Octane, 2,3,7-trimethyl, } \\
\text { Decane and N-(2-Cyano- } \\
\text { ethyl)-benzenesulfonamide }\end{array}$ & $\begin{array}{c}\eta_{\text {total }}=1.2 \% \\
\operatorname{MIC}(S . \text { aureus })=500 \mu \mathrm{g} / \mathrm{mL}\end{array}$ & [53] \\
\hline Lavandula viridis & $\mathrm{CO}_{2}$ & 40 & 120 & $0.3 \mathrm{~kg} / \mathrm{h}$ & - & - & $\begin{array}{c}\text { Antioxidant, } \\
\text { Anticholinesterase (AChE) }\end{array}$ & $\begin{array}{l}\text { Myrtenol, camphor, } \\
\text { verbenone }\end{array}$ & $\begin{array}{l}\eta_{\text {total(1st separator) }}=3.45 \% \\
\eta_{\text {total }(2 \text { nd separator })}=9.27 \%\end{array}$ & {$[54]$} \\
\hline Mentha spicata & $\mathrm{CO}_{2}$ & 60 & 200 & $15 \mathrm{~g} / \mathrm{min}$ & 60 & $3 \mathrm{~g}_{\text {ethanol }} / \mathrm{min}$ & Antioxidant & $\begin{array}{c}\text { Flavonoids (catechin, } \\
\text { epicatechin, rutin, } \\
\text { myricetin, luteolin, apigenin } \\
\text { and naringenin) }\end{array}$ & $\eta_{\text {total }}=60.57 \mathrm{mg} / \mathrm{g}$ & [55] \\
\hline Vitis labrusca & $\mathrm{CO}_{2}$ & 45 & 160 & $2 \mathrm{~mL} / \mathrm{min}$ & 30 & $\begin{array}{c}6 \% \\
\text { ethanol }\end{array}$ & Antioxidant & $\begin{array}{l}\text { Total Phenols, antioxidants } \\
\text { and Total anthocyanins }\end{array}$ & $\begin{array}{c}\eta_{\text {total }}=12.31 \% \\
\mathrm{AA}=1.628 \mathrm{mg} / \mathrm{mL} \\
\mathrm{TA}=1.17 \mathrm{mg} / \mathrm{mL} \\
\mathrm{TPC}=2.156 \mathrm{mgGAE} / 100 \mathrm{ml}\end{array}$ & {$[56]$} \\
\hline Prunus avium & $\mathrm{CO}_{2}$ & 50 & 250 & - & 150 & $\begin{array}{l}10 \% \\
\text { ethanol }\end{array}$ & $\begin{array}{l}\text { Antitumor (human colon } \\
\text { cancer cells, HT29), } \\
\text { antioxidant }\end{array}$ & $\begin{array}{c}\text { Perillyl alcohol, sakuranetin } \\
\text { and sakuranin }\end{array}$ & $\begin{array}{c}\eta_{\text {total }(1 \mathrm{st} \mathrm{step})}=0.5 \% \\
\eta_{\text {total }(2 \mathrm{nd} \mathrm{step})}=1.5 \% \\
\mathrm{TPC}=2.5 \mathrm{mgGAE} / \mathrm{g} \\
\mathrm{AA}=181.4 \mu \mathrm{mol} \mathrm{TEAC} / \mathrm{g}_{\text {dry mass }}\end{array}$ & [9] \\
\hline $\begin{array}{l}\text { Vaccinium } \\
\text { macrocarpon } \\
\text { Ait. }\end{array}$ & $\mathrm{CO}_{2}$ & 40 & $\begin{array}{l}655- \\
621\end{array}$ & $125 \mathrm{~g} / \mathrm{min}$ & - & $\begin{array}{c}0-30 \% \\
\text { (ethanol) }\end{array}$ & $\begin{array}{c}\text { Bacterial aglutination } \\
\text { (ExPEC aglutination) and } \\
\text { bacterial invasion (ExPEC } \\
\text { enterocyte invasion } \\
\text { inhibition) }\end{array}$ & Proanthocyanidins & 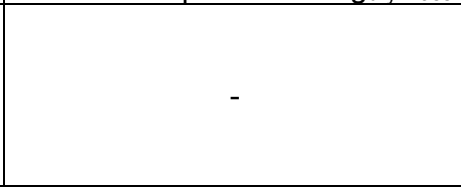 & {$[57]$} \\
\hline
\end{tabular}




\begin{tabular}{|c|c|c|c|c|c|c|c|c|c|c|}
\hline $\begin{array}{c}\text { Haematococcus } \\
\text { pluvialis }\end{array}$ & $\mathrm{CO}_{2}$ & 65 & 435 & $\begin{array}{c}166 \\
\mathrm{~mL} / \mathrm{min}\end{array}$ & 210 & $\begin{array}{c}2.3 \\
\mathrm{~mL} \text { ethanol } / \mathrm{g}_{\text {sample }} \\
\end{array}$ & Antioxidant & astaxanthin & $\eta_{\text {astaxanthin }}=87.42 \%$ & [58] \\
\hline $\begin{array}{l}\text { Nannochloropsis } \\
\text { oculata }\end{array}$ & $\mathrm{CO}_{2}$ & 50 & 350 & $10 \mathrm{ml} / \mathrm{min}$ & - & $\begin{array}{l}16.7 \% \\
\text { ethanol }\end{array}$ & Phagocytotic activity & $\begin{array}{l}\text { Zeaxanthin, triglycerides, } \\
\text { carotenoids }\end{array}$ & $\begin{array}{c}\eta_{\text {total }}=15.50 \% \\
{[\text { Triglycerides }]=239.7} \\
\mathrm{mg} / \mathrm{g}_{\text {extract }} \\
{[\text { Carotenoids }]=7.61 \mathrm{mg} / \mathrm{g}_{\text {extract }}}\end{array}$ & [59] \\
\hline Rubus sp. & $\mathrm{CO}_{2}$ & $40-60$ & $\begin{array}{l}150- \\
250\end{array}$ & $\begin{array}{l}16.62 \\
g / m i n\end{array}$ & 57 & $\begin{array}{c}5-10 \% \\
\text { ethanol/water }\end{array}$ & Antioxidant & $\begin{array}{l}\text { Anthocyanins } \\
\text { (cyanidin 3-O-glucoside, } \\
\text { cyanidin 3-O-rutinoside) }\end{array}$ & $\begin{array}{c}\eta_{\text {total }}=6.25-18.25 \% \\
{[\text { Anthocyanins }]=2.20-17.54} \\
\mathrm{mg} / \mathrm{g}_{\text {extract }}\end{array}$ & [60] \\
\hline $\begin{array}{c}\text { Piper } \\
\text { diospyrifolium }\end{array}$ & $\mathrm{CO}_{2}$ & 40 & 250 & $3 \mathrm{~mL} / \mathrm{min}$ & 120 & - & $\begin{array}{c}\text { Antibacterial } \\
(\text { Mycobacterium } \\
\left.\text { tuberculosis }\left(\mathrm{H}_{37} \mathrm{Rv}\right)\right)\end{array}$ & $\begin{array}{c}\text { 4-methoxy-3-[(E)-3-methyl- } \\
\text { 1,3-butadien-1-yl]-5-(3- } \\
\text { methyl-2-buten-1-yl)- } \\
\text { benzoic acid }\end{array}$ & $\eta_{\text {total }}=2.88 \%$ & [61] \\
\hline Bactris gasipaes & $\mathrm{CO}_{2}$ & 40 & 300 & $3 \mathrm{~L} / \mathrm{min}$ & 91 & - & Antioxidant & carotenoids & $\begin{array}{c}\eta_{\text {total }}=5 \% \\
\text { [carotenoids] }=2.01 \mathrm{mg} / \mathrm{g}_{\text {extract }}\end{array}$ & [62] \\
\hline $\begin{array}{l}\text { Melia azedarach } \\
\text { L. }\end{array}$ & $\mathrm{CO}_{2}$ & 50 & 300 & $1.65 \mathrm{~g} / \mathrm{min}$ & 180 & $\begin{array}{c}0.71 \\
\text { gethano/min }\end{array}$ & $\begin{array}{l}\text { Antiviral: HSV-1 (Herpes } \\
\text { Simplex Virus Type 1); } \\
\text { BVDV (Bovine viral } \\
\text { diarrhea virus) } \\
\end{array}$ & $\begin{array}{c}\text { Fatty acids: linoleic acid, } \\
\text { palmitic acid, myristic acid } \\
\text { Phenolics: caffeic acid, } \\
\text { malic acid } \\
\end{array}$ & $\begin{array}{c}\eta_{\mathrm{SFE}}=8.2 \% \\
\eta_{\mathrm{SFEE}}=6.4 \%\end{array}$ & [63] \\
\hline grape marc & $\mathrm{CO}_{2}$ & 40 & 80 & $4-6 \mathrm{~kg} / \mathrm{h}$ & 180 & $\begin{array}{c}7.5-10 \% \\
\text { Ethanol/water } \\
(57 \% \mathrm{v} / \mathrm{v})\end{array}$ & Antioxidant & $\begin{array}{l}\text { Proanthocyanidins, Total } \\
\text { phenolic content }\end{array}$ & $\eta_{\text {total }}=13.1-14.6 \%$ & [64] \\
\hline $\begin{array}{l}\text { Hymenaea } \\
\text { courbaril L. }\end{array}$ & $\mathrm{CO}_{2}$ & 50 & 350 & $\begin{array}{c}1.11 \mathrm{E}^{-4} \\
\mathrm{~kg} / \mathrm{s}\end{array}$ & $10_{S P}+70_{D P}$ & $\begin{array}{l}10 \% \\
\text { Water }\end{array}$ & Antioxidant & Polyphenol (procyanidins) & $\eta_{\text {total }}=24 \%$ & [65] \\
\hline Piper nigrum L. & $\mathrm{CO}_{2}$ & 40 & 300 & $2 \mathrm{~mL} / \mathrm{min}$ & $30_{S P}+40_{D P}$ & - & Antioxidant & $\begin{array}{c}\beta \text {-caryophyllene, limonene, } \\
\text { sabinene , } 3 \text {-carene, } \beta \text { - } \\
\text { pinene and } \alpha \text {-pinene. }\end{array}$ & $\eta_{\text {Total }}=2.05 \%$ & [66] \\
\hline $\begin{array}{c}\text { Melia azedarach } \\
\text { L. }\end{array}$ & $\mathrm{CO}_{2}$ & 60 & 250 & $2 \mathrm{~g} / \mathrm{min}$ & $60 \mathrm{SP}+90_{\mathrm{DP}}$ & - & Insecticidal agent & Triterpene (melianone) & $\eta_{\text {Total }}=5.15 \%$ & [67] \\
\hline $\begin{array}{l}\text { Vaccinium } \\
\text { myrtillus L. }\end{array}$ & $\mathrm{CO}_{2}$ & 40 & 200 & $1.4 \mathrm{E}^{-4} \mathrm{~kg} / \mathrm{s}$ & - & $\begin{array}{c}10 \% \\
\text { Water/ethanol } \\
(1: 1) \\
\end{array}$ & Antioxidant & Anthocyanins & $\eta_{\text {Total }}=2.7 \%$ & [68] \\
\hline $\begin{array}{c}\text { Eugenia uniflora } \\
\text { L. }\end{array}$ & $\mathrm{CO}_{2}$ & $35-55$ & $81-261$ & $2 \mathrm{~g} / \mathrm{min}$ & 360 & - & Antioxidant & $\begin{array}{l}\text { y-elemene and } \\
\text { germacrone }\end{array}$ & $\eta_{\text {Total }}=0.16-0.48 \%$ & [69] \\
\hline $\begin{array}{l}\text { Phormidium } \\
\text { valderianum }\end{array}$ & $\mathrm{CO}_{2}$ & 50 & 500 & $2 \mathrm{~L} / \mathrm{min}$ & 90 & - & $\begin{array}{l}\text { Antioxidant and anatoxin-a } \\
\text { content reduction }\end{array}$ & $\begin{array}{l}\text { Total phenolics, Total } \\
\text { carotenoids, anatoxin-a }\end{array}$ & $\eta_{\text {Total }}=3.97 \%$ & [70] \\
\hline
\end{tabular}




\begin{tabular}{|c|c|c|c|c|c|c|c|c|c|c|}
\hline Rosemary & $\mathrm{CO}_{2}$ & 40 & $\begin{array}{l}150- \\
300\end{array}$ & $60 \mathrm{~g} / \mathrm{min}$ & $60_{s 1}+120_{s 2}$ & $\begin{array}{l}0-7 \% \\
\text { ethanol }\end{array}$ & $\begin{array}{l}\text { Antitumor (HT-29 human } \\
\text { adenocarcinoma) }\end{array}$ & Carnosic acid, carnosol & $\begin{array}{l}\eta_{\text {Total }}(\mathrm{S} 1)=1.61-2.73 \% \\
\eta_{\text {Total }}(\mathrm{S} 2)=4.30-4.67 \%\end{array}$ & [5] \\
\hline $\begin{array}{l}\text { Penaeus } \\
\text { brasiliensis and } \\
\text { Penaeus } \\
\text { paulensis }\end{array}$ & $\mathrm{CO}_{2}$ & 60 & 300 & $13.3 \mathrm{~g} / \mathrm{min}$ & 180 & - & $\begin{array}{l}\text { Anti-obesity and } \\
\text { hypolipidemic }\end{array}$ & $\begin{array}{l}\text { Astaxanthin, oleic acid, } \\
\text { palmitic acid, stearic acid, } \\
\text { eicosapentaenoic acid } \\
\text { (EPA) and docosahexanoic } \\
\text { acid (DHA) from the } \omega-3 \\
\text { fatty acids group }\end{array}$ & $\begin{array}{c}\text { [palmitic acid] }=18 \% \\
{[\text { oleic acid] }=21 \%} \\
{[\text { EPA }]=11.21 \%} \\
{[\mathrm{DHA}]=7.95 \%}\end{array}$ & [71] \\
\hline $\begin{array}{l}\text { Ganoderma } \\
\text { lucidum }\end{array}$ & $\mathrm{CO}_{2}$ & 50 & 300 & $0.15 \mathrm{~kg} / \mathrm{h}$ & 210 & - & $\begin{array}{c}\text { Antitumor on CaCo-2 } \\
\text { (human colon } \\
\text { adenocarcinoma cells), } \\
\text { anticholinesterase (AChE), } \\
\text { antioxidant }\end{array}$ & $\begin{array}{l}\text { triterpenoids and } \\
\text { polysaccharides }\end{array}$ & $\eta_{\text {Total }}=1.92 \%$ & [72] \\
\hline $\begin{array}{c}\text { Thymus } \\
\text { vulgaris, } \\
\text { Thymus } \\
\text { hyemalis and } \\
\text { Thymus zygis }\end{array}$ & $\mathrm{CO}_{2}$ & 40 & 100 & - & 480 & - & $\begin{array}{l}\text { Antiviral (HSV-1) (Herpes } \\
\text { simplex virus type 1) }\end{array}$ & $\begin{array}{l}\text { Thymol, carvacrol, borneol, } \\
\text { camphor and 1,8-cineole }\end{array}$ & $\begin{array}{c}\text { thymol, carvacrol, borneol] } T_{T} . \\
\text { zygis }=79.77 \% \\
{[\text { thymol, carvacrol, borneol }]_{T} .} \\
\text { vulgaris }=73.04 \% \\
{[\text { thymol, carvacrol, borneol }]_{T} \text {. }} \\
\text { hyemalis }=19.02 \% \\
\end{array}$ & [7] \\
\hline $\begin{array}{l}\text { Hippophae } \\
\text { rhamnoides L. }\end{array}$ & $\mathrm{CO}_{2}$ & 50 & 200 & - & - & - & $\begin{array}{c}\text { Anti-inflammatory, } \\
\text { Endotoxemia inhibition }\end{array}$ & Isorhamnetin & - & [73] \\
\hline $\begin{array}{l}\text { Cyperus } \\
\text { articulatus } \mathrm{L} . \\
\text { var. articulatus }\end{array}$ & $\mathrm{CO}_{2}$ & $40-60$ & $\begin{array}{l}130- \\
250\end{array}$ & $3 \mathrm{ml} / \mathrm{min}$ & $30_{S P}+120_{D P}$ & - & $\begin{array}{c}\text { Antifungal (Cladosporium } \\
\text { sphaerospermum ATCC } \\
\text { 4464), Antibacterial } \\
\text { (Staphylococcus aureus } \\
\text { ATCC 25923) } \\
\end{array}$ & $\begin{array}{l}\text { Terpenes ( mustacone, } \alpha- \\
\text { ciperone, corimbolone and } \\
\text { cariofilene oxide) }\end{array}$ & $\eta_{\text {Total }}=1.2-3.3 \%$ & {$[74]$} \\
\hline $\begin{array}{l}\text { Pleurotus } \\
\text { ostreatus }\end{array}$ & $\mathrm{CO}_{2}$ & 48 & 210 & $20 \mathrm{~L} / \mathrm{h}$ & $10 \mathrm{sP}+80_{\mathrm{DP}}$ & $\begin{array}{l}133 \mathrm{ml} \\
\text { ethanol }\end{array}$ & Antioxidant & $\begin{array}{l}\text { Ergothioneine, total } \\
\text { phenolic content }\end{array}$ & $\begin{array}{l}\text { [ergothioneine] }=1.35 \mathrm{mg} / \mathrm{g} \\
\mathrm{TPC}=5.48 \mathrm{mg} \mathrm{g}_{\mathrm{GE}} / \mathrm{g}_{\text {extrasct }}\end{array}$ & [75] \\
\hline \multirow{2}{*}{$\begin{array}{l}\text { Eupatorium } \\
\text { intermedium }\end{array}$} & $\mathrm{CO}_{2}$ & $30-80$ & $\begin{array}{l}150- \\
250\end{array}$ & \multirow{2}{*}{$\begin{array}{c}2.0 \\
\mathrm{~cm}^{3} / \mathrm{min}\end{array}$} & \multirow{2}{*}{180} & \multirow{2}{*}{ - } & \multirow{2}{*}{$\begin{array}{c}\text { Antibacterial } \\
\text { (Staphylococcus aureus } \\
\text { and Listeria } \\
\text { monocytogenes), } \\
\text { antioxidant }\end{array}$} & \multirow{2}{*}{$\begin{array}{l}\text { spathulenol, caryophyllene } \\
\text { oxide, caryophyllene }(E), \\
\text { germacrene } D, \\
\text { bicyclogermacrene, carotol } \\
\text { and phenolic content }\end{array}$} & $\begin{array}{c}\eta_{\text {Total }}=5.85 \% \\
\mathrm{TPC}=8.74 \mathrm{mg} \mathrm{gAE}_{\mathrm{GE}} / \mathrm{g}_{\text {extrasct }} \\
\mathrm{AA}=293.47 \mathrm{mg} \alpha- \\
\text { tocopherol } / \mathrm{g}_{\text {extract }}\end{array}$ & \multirow[t]{2}{*}[76]{} \\
\hline & $\mathrm{C}_{3} \mathrm{H}_{8}$ & $20-60$ & $30-100$ & & & & & & $\begin{array}{c}\eta_{\text {Total }}=9.34 \% \\
\mathrm{TPC}=10.03 \mathrm{mg} \mathrm{GAE}_{\mathrm{GA}} / \mathrm{g}_{\text {extrasct }} \\
\mathrm{AA}=268.33 \mathrm{mg} \mathrm{a}- \\
\text { tocopherol } / \mathrm{g}_{\text {extract }}\end{array}$ & \\
\hline Salvia officinalis & $\mathrm{CO}_{2}$ & 40 & $\begin{array}{l}100_{S 1} \\
300_{S 2}\end{array}$ & $50 \mathrm{~g} / \mathrm{min}$ & 480 & - & $\begin{array}{c}\text { Anti-inflammatory (THP-1 } \\
\text { atherosclerotic) }\end{array}$ & $\begin{array}{l}\text { camphor, borneol and1,8- } \\
\text { cineole }\end{array}$ & $\begin{array}{c}\text { [camphor, borneol and1,8- } \\
\text { cineole }]_{\mathrm{S} 1}=62.4 \%\end{array}$ & [8] \\
\hline
\end{tabular}




\begin{tabular}{|c|c|c|c|c|c|c|c|c|c|c|}
\hline & & & & & & & & & $\begin{array}{c}\text { [camphor, borneol and1,8- } \\
\text { cineole }]_{S 2}=48.1 \%\end{array}$ & \\
\hline $\begin{array}{c}\text { Cordia } \\
\text { verbenacea }\end{array}$ & $\mathrm{CO}_{2}$ & 50 & 300 & $0.3 \mathrm{~kg} / \mathrm{h}$ & 270 & - & $\begin{array}{l}\text { Antitumor (COX-2 and } \\
\text { MCF-7 cells) }\end{array}$ & $\begin{array}{l}\alpha \text {-caryophyllene and } \beta \text { - } \\
\text { caryophyllene }\end{array}$ & $\eta_{\text {Total }}=5.75 \%$ & [6] \\
\hline $\begin{array}{l}\text { Chlorella } \\
\text { vulgaris }\end{array}$ & $\mathrm{CO}_{2}$ & 50 & 310 & $6 \mathrm{NL} / \mathrm{min}$ & 20 & $\begin{array}{c}50 \mathrm{ml} \\
\text { Ethanol:water } \\
(1: 1)\end{array}$ & $\begin{array}{c}\text { Antitumor (human lung } \\
\text { cancer H1299, A549 and } \\
\text { H1437) }\end{array}$ & $\begin{array}{l}\text { Total polyphenol and } \\
\text { flavonoid content }\end{array}$ & $\begin{array}{c}\eta_{\text {Total }}=8.70 \% \\
\mathrm{TPC}=13.40 \mathrm{mg} \text { GAE/gextrac } \\
\mathrm{TFC}=3.18 \mathrm{mg}_{\text {quercetin }} / \mathrm{g}_{\text {extract }}\end{array}$ & {$[77]$} \\
\hline
\end{tabular}

Abbreviations: TPC - Total phenolic content, TFC - Total flavonoid content, AA - Antioxidant activity, TA - Total anthocyanins, SFEE -Supercritical fluid extraction with ethanol as cosolvent, DP - Dynamic procedure, SP - Static procedure, S1 - First extraction step/separator; S2 - Second extraction step/separator. 
Regarding the gathered information in table 3, Benelli et al. [53] obtained extracts composed mainly of Llimonene, palmitic acid, oleic acid, $n$-butyl benzenesulfonamide and $\beta$-sitosterol (chemical profile obtained by gas chromatography coupled to mass spectrometry analysis (GC-MS)) that showed high potential as an inhibitor of microorganism growth (Staphylococcus aureus and Escherichia coli) especially at extraction conditions of $50{ }^{\circ} \mathrm{C}$ and 200 bar. Jayashankar et al. [73] applied the same extraction conditions for the extraction of Isorhamnetin (phytochemical analysis by reversed-phase high-performance liquid chromatography with a UV detector (RP-HPLC-UV)), which is a potential agent for the treatment of endotoxemia. Bitencourt et al. [78] applied different SFE conditions (50 ${ }^{\circ} \mathrm{C}, 300$ bar) with cosolvent $(30 \%$ ethanol) in order to obtain extracts with significant inhibition percentage against Herpes Simplex Virus Type 1. The extracts obtained were chemically characterized by GC-MS, which were mainly composed by fatty acids (linoleic acid, palmitic acid and myristic acid) and phenolic compounds (caffeic acid and malic acid). Serra et al. [9] used extraction conditions of $50^{\circ} \mathrm{C}, 250$ bar and $10 \%$ of ethanol (cosolvent) in order to obtain extracts that exhibited antioxidant activity and revealed human colon cancer cells growth inhibition. Upon chemical identification, perillyl alcohol was detected by thin layer chromatography (TLC) and was pointed as one of the major responsible compound for its anticancer properties. Identification and quantification of phenolics such as anthocyanins was achieved by high performance liquid chromatography with a diode-array detector (HPLC-DAD), whereas sakuranin and sakuranetin compounds were identified by liquid chromatography with a diode-array detector and tandem mass spectrometry (LCDAD-MS/MS), which were indicated as the major contributors of its antioxidant capacity.

\subsection{Operative conditions}

One of the most appreciated advantages of SFE is the low critical temperature of one of its most applied fluids, the $\mathrm{CO}_{2}$. A low extraction temperature is of key importance for the preservation of such bioactive compounds in the extracts. In order to achieve higher global/specific yields or higher bioactivity capabilities (e.g. antitumor, antimicrobial, antibacterial, antiviral and anti-inflammatory), $43 \%$ of the work presented in Table 3 applies an extraction temperature range of $40{ }^{\circ} \mathrm{C}$ to $50{ }^{\circ} \mathrm{C}$, followed by $33 \%$ at from $50{ }^{\circ} \mathrm{C}$ to 60 ${ }^{\circ} \mathrm{C}$. Regarding the pressure trend applied in the works presented it Table 3, 37\% employed a pressure range of 200 bar to 300 bar followed by $28 \%$ from 300 bar to 400 bar.

While solute vapor pressure is only temperature-dependent, pressure and temperature can be physically related in terms of density, viscosity and diffusivity. When variations of temperature and/or pressure are considered, these have major repercussions regarding fluid hydrodynamics, solubility and mass transfer, thus defining in great extent the performance of a SFE system. Moreover, when at constant pressure and temperature suffers a variation, this action results in contrary effects upon solubility, since $\mathrm{SC}-\mathrm{CO}_{2}$ density and solute vapor pressure exhibit conflicting behaviors (when temperature increases, density decreases and vapor pressure of solute increases).

Bimakr et al. [55] obtained bioactive flavonoid compounds from spearmint (Mentha spicata L.) leaves by performing an experimental design study (complete randomized full factorial design) of the effects of different temperatures $\left(40,50\right.$ and $\left.60{ }^{\circ} \mathrm{C}\right)$, pressures $(100,200$ and 300 bar) and time (30,60 and 90 min) in order to find the optimal SFE conditions which were $60{ }^{\circ} \mathrm{C}, 200$ bar and $90 \mathrm{~min}$. According to Bimakr et al. [55], the effect of a temperature increase on solute solubility was considered as the main reason why the flavonoid yield changed significantly over the temperature range of $40-60{ }^{\circ} \mathrm{C}$.

Regarding the pressure effect on the extraction, an increase of pressure can result in an increase in fluid density altering solute solubility, which according to Gomes et al. [79] a higher recovery of volatile fractions and a lower recovery of non-volatile fractions are obtained at high pressures. Therefore, it is of interest to control the composition of the extract using pressure. Bimakr et al. [55] increased the flavonoid yield with 
increasing pressure to a certain value (200 bar). However, over this pressure range, a decrease in flavonoid yield was observed and the volatility of the extracts could be held accountable for such outcome. Another example is the extraction of bioactive compounds with antioxidant activity from peach palm pulp (Bactris gaspaes) performed by Espinosa-Pardo et al. [58] who studied different extraction condition, namely temperature $\left(40,50\right.$ and $\left.60^{\circ} \mathrm{C}\right)$ and pressure $(100,200$ and 300 bar). In this particular case, the optimal conditions for the extraction of carotenoids were of $40{ }^{\circ} \mathrm{C}$ and 300 bar. These differences between optimal temperature and pressure conditions strongly depend on the biomass origin, morphological part and condition, as well as on the targeted compound to be extracted.

The proportion and the type of modifier are also of extreme importance within the extraction itself. Both are key factors on the solubility of the target compounds in the supercritical fluid extraction. In this review, $47 \%$ of the works presented in Table 3 use modifiers in order to enhance the extraction of their targeted bioactive compounds, being ethanol the most common applied modifier with a proportion that can vary from $5 \%$ to $30 \%$. Roseiro

[52] discovered that the optimal SFE conditions in order to obtain the highest phenolic concentration and antioxidant capacity from carob kibbles (Ceratonia silique) with antiproliferative activity on rat N1E-115 neuroblastoma cells, and on human HeLa cervical and MCF-7 breast cancer cell lines were 220 bar, $40{ }^{\circ} \mathrm{C}$ with a $10 \%$ cosolvent mixture composed by $80 \%$ ethanol and $20 \%$ water, revealing that carob SFE extract possessed a great potential as a source of natural antitumor compounds. The authors [52] also compared the efficiency of SFE with ultrasound extraction and conventional extraction and concluded that for the conventional and ultrasound extractions the more efficient extraction was obtained with $70 \%$ aqueous acetone rather than with water, regardless the extraction methods and conditions. The total phenolics content has been 14.7, 20.4 and $27.1 \mathrm{mg} \mathrm{GAE} / \mathrm{g}$ dry mass while the antioxidant activity has been 0.027 , 0.030 and $0.130 \mathrm{mmol} \mathrm{TEAC} / \mathrm{g}$ dry mass, respectively for conventional, ultrasound and SFE extractions. These results showed that the higher antioxidant capacity (four times) has been obtained with SFE. This can be explained by the different phenolic profiles of the extracts obtained with each extraction technique.

Bhattacharya et al. [75] also demonstrated that pressure and the amount of cosolvent were the most effective parameters on their system response, and that the optimal conditions to obtain the highest ergothioneine and polyphenols concentration from Pleurotus ostreatus along with the highest antioxidant capacity were 210 bar, $48^{\circ} \mathrm{C}$ with an ethanol amount of $133 \mathrm{ml}$.

An alternative to $\mathrm{CO}_{2}$ in supercritical extractions is the use of propane. Although propane does not offer many of the qualities that are commonly associated with $\mathrm{CO}_{2}$, this reasonably inexpensive solvent can be a better choice for the extraction of oils and natural products. Propane does not leave a toxic residue just as $\mathrm{CO}_{2}$ but the required extraction pressures are inferior to those applied with $\mathrm{scCO}_{2}$ [80].

Czaikoski et al. [76] performed SFE studies that involved $\mathrm{CO}_{2}$ and propane as a supercritical solvents on the extraction of bioactive compounds from Eupatorium intermedium, and they observed that when $\mathrm{scCO}_{2}$ was applied, pressure had a positive effect on the extraction yield while with propane, both pressure and temperature had a positive effect on the final yield. Furthermore, antibacterial tests indicated that different bacterial species exhibited different levels of sensitivity towards the $E$. intermedium extract. Both of the Gram-negative bacteria tested, Escherichia coli and Salmonella typhimurium, were completely resistant to the extracts obtained using $\mathrm{scCO}_{2}$ and compressed propane. However, the Gram-positive bacteria evaluated, Staphylococcus aureus and Listeria monocytogenes, were susceptible to the same extracts indicating their potential application as natural preservatives against food-borne pathogens. 
Porto et al. [49] compared water and ethanol as cosolvent in SFE of proanthocyanidins from grap marc. Considering the polarity of polyphenols, the addition of a modifier (cosolvent) was needed. The authors compared several operative conditions such as solvent $\mathrm{CO} 2$, water and ethanol $15 \%(\mathrm{w} / \mathrm{w})$, pressure of 100 and $200 \mathrm{MPa}$, and temperatures of $313.15,323.15$ and $333.15 \mathrm{~K}$. The performance of the methodology was verified by evaluation of phenolic yield, proanthocyanidins content and antioxidant activity. Three methodologies were tested SC-CO2 plus water, SC-CO2 plus ethanol, and SC-CO2 plus water followed by SC-CO2 plus ethanol and the global yields were 10.2,6.9 and $10.8 \%$ (w/w), respectively. The extraction yields obtained by SFE were lower than classical extraction with methanol $(15.6 \%(\mathrm{w} / \mathrm{w}))$. The phenolic yields obtained were $63.4 \mathrm{~g} / \mathrm{kg}, 38.8 \mathrm{~g} / \mathrm{kg}$ and $68.0 \mathrm{~g} / \mathrm{kg}$ of extract for SC$\mathrm{CO}_{2}+15 \% \mathrm{~W}$, of extract for $\mathrm{SC}-\mathrm{CO}_{2}+15 \% \mathrm{EtOH}$, and $\mathrm{Sc}-\mathrm{CO}_{2}+15 \% \mathrm{~W}$ extraction, followed by SC$\mathrm{CO}_{2}+15 \% \mathrm{EtOH}$, respectively. This last procedure gave the highest phenolic yield (68.0 g/kg of extract), phenol content (733.6 mg GAE/100 g DM), proanthocyanidins concentration (572.8 mg catechin/100 g $\mathrm{DM}$ ) and antioxidant activity (2649.6 mg $\alpha$-tocopherol/100 g DM). Although the classical methanolic extraction gives highest phenolic yield (180.3 g/kg of extract) and phenol content (2813.2 mg GAE/100 g $\mathrm{DM})$ the same thus not applies to the antioxidant activity (677.9 mg a-tocopherol/100 $\mathrm{g} \mathrm{DM}$ ) that suggests that SFE allow the extraction of different phenol fractions responsible for the high antioxidant activity of the extracts.

In another study Porto et al [63] verified the effect of solvent (CO2) flow rate (4 and $6 \mathrm{~kg} / \mathrm{h} \mathrm{CO}_{2}$ ) on the extraction of total phenols from grape marc and verified that the mass transfer increases with the decrease in flow rate. This could be attributed to fact that the contact time between the solvent and the compounds to be extracted increased with the lower flow rate, thus affecting positively the extraction efficiency.

\subsection{Technological advancements and extraction efficiency}

Regarding the efficiency of extraction, biomass particle size, shape and porosity has a direct relationship with the extraction itself, thus becoming an important factor in the mass-transfer rate $[40,81]$. Although the usage of small particles size promotes the increase of surface area, leading to the increase of accessible solute, they can lead to the obstruction of the extraction, rendering the use of dispersive agents useful in such cases. Therefore, particle size condition is of great influence on the extraction yield [2,81]. The choice of optimal extraction conditions also demands concrete and pristine knowledge regarding the targeted biomass matrix, as well as the solubility of the targeted compounds to be extracted by SFE technique regardless of their nature. In addition to particle size, shape, and porosity of the matrix itself, there are other important factors to consider; Genetics, biomass maturity, different morphological parts of the biomass, morphological location of the solute in the matrix and the impact of climate conditions and nutrients on the biomass growth are all conditions that can have a profound impact on the extraction yield and quality of the extract [40].

In order to improve extraction efficiency, several approaches have been deemed useful to the SFE procedure itself. From the biomass pretreatment, with enzymes, basic modifiers, ionic liquids, or even with sudden supercritical $\mathrm{CO}_{2}$ decompression, to the combination of other technologies such as ultrasound and enzymes with SFE, there are several approaches that can be performed in order to enhance the extraction yield and the selectivity of the targeted bioactive compounds themselves.

In recent years ionic liquids (ILs) have received much attention as a result of their special and unique properties, which can be used advantageously in green analytical chemistry as an alternative to organic solvents in the separation and improvement of analysis of added-value components extracted from biomass $[82,83]$. ILs which are salts with low melting points, constituted by large and asymmetric organic 
cations and organic or inorganic anions being liquid at/or near room temperature, can have their polarities and affinities tailored by a proper manipulation of the cation/anion design and thus allowing a large spectrum of bioactive compound dissolution diversity [83-85]. These solvents have excellent properties (negligible vapor pressure, thermal stability, tunable viscosity, miscibility with water and organic solvents and favorable solvating properties for a wide spectrum of polar and non-polar compounds) and have been proposed as environmentally friendly solvents for "green chemistry" due to their ability to substitutes traditional organic solvents. Nevertheless, this "green" label as raised some controversy because of their incomplete physical, chemical and toxicological data [2]. ILs in analytical chemistry are mainly focused on their applicability as solvents for extraction in conventional solid-liquid extraction, microwave-assisted extraction and ultrasonic-assisted extraction [83].

Although the low volatility of ILs is one of the key aspects that makes them green solvents, this advantage also represents a problem for product separation and recovery. Several techniques for the recovery of volatile products from ILs can be accomplished by distillation or evaporation, however, when faced with non-volatile or thermo-sensitive products these methods cannot be considered. Hydrophobic ILs can be extracted with water to separate water-soluble solutes, nonetheless, this method is not suited for hydrophilic ILs and, although organic solvents could be applied in such cases, this approach would compromise the goal for "green chemistry" and lead to cross contamination between both phases. Bearing this in mind, supercritical fluids emerges here, and once more, as an alternative green method that can solve all of the abovementioned predicaments while recovering various types of solutes [86].

Teixeira [87] shows that it is possible to achieve the dissolution and hydrolysis of wet algae by the use of ILs without acids, bases or catalysts, as a cost and energy-efficient process in order to succeed in the deconstruction of the algae cell walls in order to separate lipids. Hierro et al. [88] states that ILs remove the cell wall and solubilize all cell contents except for lipids, which are virtually immiscible, and suggests that the extraction of the lipids could be accomplished by the use of $\mathrm{scCO}_{2}$. ILs can therefore be integrated in the SFE, by being accurately designed to either assist the extraction itself by improving the extraction selectivity and efficiency and therefore be used as a cosolvent, or be applied as a pretreatment agent for the biomass. However, the combination of $\mathrm{scCO}_{2}$ with ILs has so far been limited to its application as a recovery agent of compounds from ILs by using $\mathrm{scCO}_{2}$ as a cosolvent or as an anti-solvent in precipitations $[89,90]$ thus further exploratory applications of SFE with ILs such as their use as cosolvent or as a pretreatment agent should be considered.

Regarding other technological improvements, the use of enzyme-assisted extraction (EAE) combined with SFE has only been marginally explored as a form of biomass pretreatment. It consists on the use of enzymatic mixtures as a mean to promote cell wall hydrolysis deconstruction leading to an increased accessibility of the supercritical solvent to the matrix solute, thus increasing the extraction yield and selectivity [2,91].

In order to achieve higher yields and selectivity of alkaloids extracts, the use of polar modifiers becomes a requisite due to the alkaloids medium to high polarity nature. Beside modifiers being required in supercritical $\mathrm{CO}_{2}$ extractions of such targeted compounds, Rachmaniah et al. [92] increased its efficiency by the usage of a basic modifier. Because alkaloids are mostly present in plant biomass as salts and not as free bases, they used a biomass pretreatment with a basic solution in order to convert the protonated salt to its non-protonated free bases, thus improving their $\mathrm{CO}_{2}$ solubility and ultimately, their extraction yield [92].

The use of sudden supercritical $\mathrm{CO}_{2}$ decompression as proposed by Fu et al. [93] and also used by Cör et al. [72] before dynamic extraction occurs, proved to be an essential step for the isolation of biological active compounds. Bioactive compounds in fungus are mainly located in the mycelium and fruiting body 
(spores). The sudden decompression stimulates the rupture of cell walls, thus providing higher accessibility of the solvent to the solute, increasing mass transfer rates that translate into higher yields and selectivity.

The application of high intensity ultrasound as a technological improvement that can be combined with SFE, comprises the control of frequency and power level which will lead to the formation and control of cavitation bubbles and repeated cycles of expansion and compression in the medium. Since bubble size is frequency dependent, higher frequencies produce smaller and less energetic bubbles which imply higher power level input in order to guarantee a good homogeneity inside the extraction vessel. These ultrasound cycles may lead to the rupture of cell walls of a biomass matrix substrate, favoring the penetration of the solvent and mass transfer. Thus, ultrasound has been used to provide higher yields and reduce extraction times. However, when a fluid is bound to high pressures, a natural limitation rises on the application of ultrasound due to the acoustic intensity required to produce cavitation [60].

Reátegui et al. [60] used ultrasound in the extraction of antioxidant compounds from blackberry bagasse with SFE and observed an increase up to $30 \%$ in global yield when $200 \mathrm{~W}$ of ultrasound was applied to the extraction conditions of $150 \mathrm{bar}, 40^{\circ} \mathrm{C}$, while at conditions of $200 \mathrm{bar}$ and $50^{\circ} \mathrm{C}$ the yield increment was only of $5 \%$. These differences in total yield gains at different operative extraction pressures can be associated, as abovementioned with the existence of a natural limitation to the ultrasound application when it is combined with high fluid pressures. These results clearly showed that coupling ultrasound with SFE will improve extraction yields, reducing the required time and operational cost.

Sample collection procedures have also evolved. Traditionally, extracts were obtained using collection vessels where the extracts precipitate after $\mathrm{CO}_{2}$ is depressurized and vented through an exhaustion pipe. This action can result in a loss of yield, mainly volatile compounds that escape with the vented $\mathrm{CO}_{2}$. In light of such occurrences, new trapping solution have been suggested and implemented. Furthermore, for the correct selection of the appropriate trapping methodology (e.g., solid trapping, liquid trapping or their combination), it is crucial to take into account the analyte volatility and polarity, the volatility of the extracting solvent, solvent modifier, and solvent flow rate [2].

In order to further increase the process selectivity, fractionation operations can be applied in the extraction and/or separation steps with two or more modules respectively. This system can be considered useful when there are numerous chemicals to be extracted separately from the same biomass matrix. There are two different approaches to the fractionation operation, a single stage where the separation step is performed on sequential separators operating at different conditions, and a two-step procedure. In the latter, the first extraction step occurs at low solvent density while in the second step the extraction occurs in a solvent at higher density, thus favoring the extraction of heavier compounds [40]. S. Santoyo et al. [7] and E. Arranz et al. [8] resorted to the use of two different extract fractionation in order to produce two extracts with different compositions in each collection vessel. The use of two different fractionation pressures allowed a gradual precipitation of the extracted compound based on their solubility in the solvent. The first vessel which was bound to higher decompression values (10 MPa) was enriched with higher weight precipitate, while the second vessel was rich in lower molecular (essential oils) weight extracts. This is particularly important since according to Koch et al. [94] antiviral activity of several plant species have been related to their essential oils, thus, rendering the separation of rich antiviral extract, with lower molecular weight, from other compounds of upmost importance.

\subsection{Design of experiments for optimization of SFE}


The design of experiments (DOE) or experimental design approach can provide a systematic investigation route as well as sequential steps for understanding linear and more complex types of interaction. The main goal of experimental design on SFE is to optimize the response variables of a certain sample by systematic modification of the controlling variables (e.g. temperature, pressure and time), while rejecting the inconsistency in the responses due to uncontrolled noisy variables in the experiment. Moreover, the selection of experimental design for SFE depends on the experimental focus, feasibility, time consumption, as well as many other relevant factors. For instance, a two level factorial design can be selected to screen the most significant factors, whereas to achieve optimization of such significant factors, more complex designs like Central Composite or Box-Behnken design is required. Therefore, experimental designs can be categorized into two groups: screening and optimization design [24,95].

Although SFE can be influenced by a large number of factors, only some have a dominant effect over the extraction. The search of these main factors marks the screening process as the main objective for the implementation of experimental design in SFE where two-level full factorial, two-level fractional factorial and Plackett-Burman design are often used for such purposes. Scapinello et al. [67] applied a two variable and two level full factorial experimental design in order to screen the most significant effect between temperature and pressure, and through the analysis of variance, determined that only pressure had significant influence on the extraction yield, and that extraction temperature and the cross effect of temperature and pressure had no such influence.

Additionally, the optimization process of experimental design which is used to discover the optimal conditions or settings for the SFE, usually starts with a screening design to select the important factors and evolves to an optimization design where Taguchi, central composite and Box-Behnken are the most applied forms [24]. Bagheri et al. [66] applied a central composite experimental design with response surface methodology with three variables (temperature, pressure and time) and two levels, performing a total of 20 extraction in order to optimize the antioxidant activity, where pressure and extraction time were the most and the least significant studied variables respectively. Reátegui et al. [60] on the other hand applied a Box-Behnken experimental design with three variables (temperature, pressure and ultrasound power) and three levels, performing a total of 15 extractions in order to attain optimal global extraction yield, anthocyanins concentration, total phenolic content and antioxidant activity.

Therefore, experimental design of SFE can prove to be a powerful research tool as screening and optimization of bioactive compounds is mandatory in order to achieve higher total/partial yield or optimal values of antitumor, antimicrobial, antibacterial, antiviral and anti-inflammatory activity.

\section{Structural characterization of bioactive compounds}

The process of structural determination involves data collection from diverse and numerous sources, each contributing with a partial view which will ultimately by integration provide unequivocal and rigorous information about the investigated chemical structure. This chemical characterization can be accomplished by several analytical technologies that do not require rigorous purification. However, in some cases, collected data is insufficient and does not allow an unequivocal confirmation of the compound structure, thus requiring a higher level of purification by techniques such as recrystallization, sublimation or distillation in order to provide rigorous structural determination [15].

The basis of the current modern structural analysis is a wide range of spectroscopic instrumentation such as ultraviolet (UV)/visible (Vis) and infrared (IR) absorption spectroscopies, nuclear magnetic resonance (NMR) spectroscopy, and mass spectrometry (MS). The development and remarkable improvements in hyphenated analytical methods over the last two decades (e.g. GC-MS, HPLC-MS, HPLC-NMR, HPLC- 
DAD-MS-NMR, ESI-MS, UPLC-QTOF-MS) have significantly broadened their application in the analysis of natural products, by increasing the speed and sensitivity of structure determinations [15,96].

Whenever the spectroscopic analysis data of an unknown compound is dubious, and the obtained compound or its derivatives is crystalline, a single crystal X-ray diffraction should be performed. The correct interpretation of such technique can provide valuable information on the three dimensional shape of the molecule, bond lengths and angles, and possible intra and intermolecular interactions, including the relative, and in some cases, the absolute stereochemistry. Nevertheless, it is important to realize that this is solid-state data and cannot give viable information about the compound in solution where properties such as hydrogen bonding may show significant effects. Since enantiomeric structures can have entirely different biological properties it is highly important to determine the absolute stereochemical nature of such structures [15].

Liau et al. [59] extracted lipids and carotenoids from microalgae (Nannochloropsis oculata) and showed that continuous modification of supercritical carbon dioxide with ethanol as cosolvent was highly important in order to achieve high extraction efficiency of such compounds. In this study SFE conditions of 350 bar, $323 \mathrm{~K}$ and $16.7 \mathrm{wt} \%$ of ethanol, presented a total yield value of $15.5 \%$. Therefore the use of ethanol as a solvent modifier presented higher extraction selectivity of carotenoid compounds presenting a recovery of $74.7 \%$ against $70.3 \%$ when compared with the ethanol soxhlet extraction counterpart. In this work, the triglycerides quantification was performed in a gas chromatography equipment with a flame ionization detector (GC-FID), while the carotenoids quantification was accomplished in a high-performance liquid chromatography with a UV detector (HPLC-UV).

To achieve the separation and purification of the carotenoid Zeaxanthin, two different approaches were explored, the fractionation of carotenoids and the use of supercritical carbon dioxide as an anti-solvent. The fractionation of carotenoids was achieved by a Soxhlet extraction with dichloromethane followed by chromatography using a silica gel column. Further purification (Zeaxanthin purity of 93.8\%) was performed by a gradient reverse-phase HPLC (RP-HPLC), followed by analysis and quantification by HPLC-UV and ${ }^{1} \mathrm{H}$ NMR analysis.

With the anti-solvent supercritical carbon dioxide approach, Liau et al. [59] injected a sample containing $40 \%$ Zeaxanthin into supercritical $\mathrm{CO}_{2}$ which resulted in a large decrease in solution density, leading to a reduction in solubility of the solid and precipitation, thus further enhancing the Zeaxanthin purity from $40 \%$ to $67.4 \%$. Liau et al. [59] states that although purity value of $93.8 \%$ Zeaxanthin was obtained by column chromatography, this process is time and solvent consuming while the anti-solvent process can generate purified solids containing $67.4 \%$ of Zeaxanthin within minutes.

Regarding the bioactivity present in the extracts, SFE with ethanol revealed lower phagocytotic activity when compared with extraction without cosolvent. However, ultrasonic water samples of the SFE with cosolvent defatted the extracts and displayed the higher phagocytotic activity recorded.

\section{Conclusions and future trends}

Supercritical fluid extraction technology can offer attractive features for obtaining bioactive compounds and overcome many limitations that exist in other extraction methodologies. Due to their enhanced transport properties, SFE allows the control of fluid density by changing its pressure and/or temperature thus providing faster extraction rates. Carbon dioxide is the most used supercritical since it is innocuous, 
preserves the extracts from atmospheric oxidation and has a low critical temperature which is of key importance for the preservation of bioactive compounds in the extracts.

The bioactivities from natural compounds obtained by SFE were mainly antioxidant (41\%), antitumor (18\%) and antibacterial activity $(10 \%)$, followed by antiviral, antimicrobial, anti-inflammatory and anticholinesterase (in a total of $5 \%$ ). In order to achieve a higher global/specific yield or higher bioactivity capabilities, $43 \%$ of the work applied an extraction temperature range of 40 to $50{ }^{\circ} \mathrm{C}$, followed by $33 \%$ at 50 to $60{ }^{\circ} \mathrm{C}$, while the pressure trend was $37 \%$ for a pressure range of 200 to 300 bar followed by $28 \%$ at 300 to 400 bar. The proportion and the type of modifier also have an important role on the extraction, allowing the manipulation of the solubility of the target compounds in the supercritical fluid extraction. Consequently, $47 \%$ of the reviewed works applied modifiers in order to enhance the extraction of their targeted bioactive compounds, where ethanol was the modifier of choice with a proportion that could vary between $5 \%$ and $30 \%$.

In order to further enhance the extraction yield and the selectivity of the targeted bioactive compounds, several technological advancements have been performed in combination with SFE methodology. From biomass pretreatment with enzymes, which promote cell wall hydrolysis, leading to higher extraction yields and selectivity due to higher mass transfer rates promoted by the ease of solvent penetration in the biomass matrix; the use of ionic liquids which can be designed to achieve multiple purposes such as the dissolution of cell walls and to attain better dissolution of the targeted bioactive compounds, being the SFE applied for the removal of these compounds from the ILs; the use of basic modifiers which convert protonated salt to their non-protonated free bases and lead to a higher $\mathrm{CO}_{2}$ solubility thus increasing their solubility, which translates in higher selectivity and yields; the use of the sudden supercritical $\mathrm{CO}_{2}$ decompression as a pretreatment operation which promotes the rupture of the biomass cell walls contributing to better solvent penetration which in consequence increase the ease of access of the solute by the solvent providing higher selectivity and yields; to the combination with other technologies such as ultrasound that triggers the rupture of the biomass cell walls through the cavitation effect leading to an increased solvent penetration thus favoring higher extraction yields. Despite the vast number of SFE applications regarding the combination of different technologies in order to achieve higher extraction efficiency there still is margin for further improvements, and additional combinations with all the abovementioned enhancements should be considered. Accordingly, it is expected that the integration of single and combined technologies will lead to higher extraction yields and greater selectivity of such bioactive compounds with significant interest to the pharmaceutical industry.

Experimental design has been also a powerful aid for screening and optimization of variables affecting the extraction of bioactive compounds, becoming mandatory in order to achieve higher total/partial yield and optimal values of antitumor, antimicrobial, antibacterial, antiviral and anti-inflammatory activities. Furthermore, SFE technique and its applied optimization methodologies are highly interconnected with several advanced molecular structure characterization techniques, becoming a valuable tool not only for the characterization and quantification of the extracts (e.g. HPLC-UV and GC-MS) but also for the purification process (RP-HPLC) of some high interest compounds.

This critical review highlighted and discussed the advantages of SFE as an alternative to conventional extraction techniques such as Soxhlet extraction. SFE can therefore be regarded as a more sustainable, cleaner and environmental friendly extraction process in the research of bioactive compounds, while providing tools and technology output for future laboratorial and industrial development.

\section{Acknowledgements}


This work was supported by European Funds through COMPETE and by National Funds through the FCT under project UID/AMB/50017/2013 and also by National funds through FCT/MEC (PIDDAC) under project IF/00407/2013/CP1162/CT0023.

\section{References}

[1] D. Mišić, I. Zizovic, M. Stamenić, R. Ašanin, M. Ristić, S.D. Petrović, D. Skala, Antimicrobial activity of celery fruit isolates and SFE process modeling, Biochem. Eng. J. 42 (2008) 148-152.

[2] M. Herrero, M. Castro-Puyana, J.A. Mendiola, E. Ibañez, Compressed fluids for the extraction of bioactive compounds, Trends Anal. Chem. 43 (2013) 67-83.

[3] M. Herrero, A. del Pilar Sánchez-Camargo, A. Cifuentes, E. Ibáñez, Plants, seaweeds, microalgae and food by-products as natural sources of functional ingredients obtained using pressurized liquid extraction and supercritical fluid extraction, Trends Anal. Chem. (2015) 1-13.

[4] J.L. Martinez, ed., Supercritical Fluid Extraction of Nutraceuticals and Bioactive Compounds, illustrate, CRC Press, 2008.

[5] A.D.P. Sánchez-Camargo, A. Valdés, G. Sullini, V. García-Cañas, A. Cifuentes, E. Ibáñez, M. Herrero, Two-step sequential supercritical fluid extracts from rosemary with enhanced antiproliferative activity, J. Funct. Foods. 11 (2014) 293-303.

[6] E.B. Parisotto, E.M.Z. Michielin, F. Biscaro, S.R.S. Ferreira, D.W. Filho, R.C. Pedrosa, The antitumor activity of extracts from Cordia verbenacea D.C. obtained by supercritical fluid extraction, J. Supercrit. Fluids. 61 (2012) 101-107.

[7] S. Santoyo, L. Jaime, M.R. García-Risco, M. Lopez-Hazas, G. Reglero, Supercritical fluid extraction as an alternative process to obtain antiviral agents from thyme species, Ind. Crops Prod. 52 (2014) 475-480.

[8] E. Arranz, L. Jaime, M.C. Lopez de la Hazas, G. Vicente, G. Reglero, S. Santoyo, Supercritical sage extracts as anti-inflammatory food ingredients, Ind. Crops Prod. 54 (2014) 159-166.

[9] A.T. Serra, I.J. Seabra, M.E.M. Braga, M.R. Bronze, H.C. de Sousa, C.M.M. Duarte, Processing cherries (Prunus avium) using supercritical fluid technology. Part 1: Recovery of extract fractions rich in bioactive compounds, J. Supercrit. Fluids. 55 (2010) 184-191.

[10] H.H. Kashani, E.S. Hoseini, H. Nikzad, M.H. Aarabi, Pharmacological properties of medicinal herbs by focus on secondary metabolites, Life Sci. J. 9 (2012) 509-520.

[11] K.G. Ramawat, S. Dass, M. Mathur, The Chemical Diversity of Bioactive Molecules and Therapeutic Potential of Medicinal Plants, in: K.G. Ramawat (Ed.), Herbal Drugs: Ethnomedicine to Modern Medicine, Springer, 2009: pp. 7-32.

[12] K. Bone, S. Mills, Principles of herbal pharmacology, in: Principles and Practice of Phytherapy: Modern Herbal Medicine., 2nd ed., Elsevier, 2013: pp. 17-82.

[13] M. Heinrich, J. Barnes, S. Gibbons, E.M. Williamson, Importance of plants in modern pharmacy and medicine, in: Fundamentals of Pharmacognosy and Phytherapy, 2nd ed., Elsevier, 2012: pp. $3-9$. 
[14] P.M. Dewick, Secondary metabolism: The building blocks and construction mechanisms, in: Medicinal Natural Products: A Biosyntethetic Approach, 2nd ed., Wiley, 2010: pp. 7-34.

[15] S.M. Colegate, R.J. Molyneux, An introduction and overview, in: S.M. Colegate, R.J. Molyneux (Eds.), Bioactive Natural Products: Detection Isolation and Structutural Determination, 2nd ed., CRC Press, 2011: pp. 1-9.

[16] S.D. Sarker, Z. Latif, A.I. Gray, eds., Natural Products Isolation, 2 Edition, Humana Press Inc., New Jersey, 2006.

[17] V. Camel, Recent extraction techniques for solid matrices-supercritical fluid extraction, pressurized fluid extraction and microwave-assisted extraction: their potential and pitfalls, Analyst. 126 (2001) 1182-1193.

[18] J. Lindy, ed., Supercritical Fluid Extraction (Technology, Applications and Limitations), Nova Science Publishers, Inc., New York, 2014.

[19] A. Pandey, S. Tripathi, Concept of standardization, extraction and pre phytochemical screening strategies for herbal drug, J. Pharmacogn. Phytochem. 2 (2014) 115-119.

[20] M. Al-jabari, Kinetic models of supercritical fluid extraction, J. Sep. Sci. 25 (2002) 477-489.

[21] J.R. Pedersen, J.O. Olsson, Soxhlet extraction of acrylamide from potato chips, Analyst. 128 (2003) 332-334.

[22] F. Chemat, M.A. Vian, G. Cravotto, Green extraction of natural products: Concept and principles, Int. J. Mol. Sci. 13 (2012) 8615-8627.

[23] J. Sunarso, S. Ismadji, Decontamination of hazardous substances from solid matrices and liquids using supercritical fluids extraction: A review, J. Hazard. Mater. 161 (2009) 1-20.

[24] K.M. Sharif, M.M. Rahman, J. Azmir, A. Mohamed, M.H. a Jahurul, F. Sahena, I.S.M. Zaidul, Experimental design of supercritical fluid extraction - A review, J. Food Eng. 124 (2014) 105-116.

[25] E.L.G. Oliveira, A.J.D. Silvestre, C.M. Silva, Review of kinetic models for supercritical fluid extraction, Chem. Eng. Res. Des. 89 (2011) 1104-1117.

[26] M. Herrero, A. Cifuentes, E. Ibañez, Sub- and supercritical fluid extraction of functional ingredients from different natural sources: Plants, food-by-products, algae and microalgae, Food Chem. 98 (2006) 136-148.

[27] M. Zougagh, M. Valcárcel, A. Ríos, Supercritical fluid extraction: A critical review of its analytical usefulness, Trends Anal. Chem. 23 (2004) 399-405.

[28] Q. Lang, C.M. Wai, Supercritical fluid extraction in herbal and natural product studies - A practical review, Talanta. 53 (2001) 771-782.

[29] M. Hierro, Supercritical fluid extraction of vegetable and animal fats with $\mathrm{CO}_{2}-\mathrm{A}$ mini review, Food Chem. 45 (1992) 189-192.

[30] A. Staby, J. Mollerup, Separation of constituents of fish oil using supercritical fluids: a review of experimental solubility, extraction, and chromatographic data, Fluid Phase Equilib. 91 (1993) 349386. 
[31] H.-W. Yen, S.-C. Yang, C.-H. Chen, Jesisca, J.-S. Chang, Supercritical fluid extraction of valuable compounds from microalgal biomass, Bioresour. Technol. 184 (2015) 291-296.

[32] C.M. Wai, S. Wang, Supercritical fluid extraction: Metals as complexes, J. Chromatogr. A. 785 (1997) 369-383.

[33] M.M.R. de Melo, A.J.D. Silvestre, C.M. Silva, Supercritical fluid extraction of vegetable matrices: Applications, trends and future perspectives of a convincing green technology, J. Supercrit. Fluids. 92 (2014) 115-176.

[34] Z. Huang, X.-H. Shi, W.-J. Jiang, Theoretical models for supercritical fluid extraction, J. Chromatogr. A. 1250 (2012) 2-26.

[35] H. Sovová, Modeling the supercritical fluid extraction of essential oils from plant materials., J. Chromatogr. A. 1250 (2012) 27-33.

[36] M. Herrero, J. a Mendiola, A. Cifuentes, E. Ibáñez, Supercritical fluid extraction: Recent advances and applications., J. Chromatogr. A. 1217 (2010) 2495-511.

[37] Ž. Knez, E. Markočič, M. Leitgeb, M. Primožič, M. Knez Hrnčič, M. Škerget, Industrial applications of supercritical fluids: A review, Energy. 77 (2013) 235-243.

[38] S.M. Pourmortazavi, S.S. Hajimirsadeghi, Supercritical fluid extraction in plant essential and volatile oil analysis, J. Chromatogr. A. 1163 (2007) 2-24.

[39] M.N. Sovilj, B.G. Nikolovski, M.D. Spasojević, Critical review of supercritical fluid extraction of selected spice plant materials, Maced. J. Chem. Chem. Eng. 30 (2011) 197-220.

[40] C.G. Pereira, M.A. a. Meireles, Supercritical Fluid Extraction of Bioactive Compounds: Fundamentals, Applications and Economic Perspectives, Food Bioprocess Technol. 3 (2010) 340372.

[41] A. Capuzzo, M. Maffei, A. Occhipinti, Supercritical Fluid Extraction of Plant Flavors and Fragrances, Molecules. 18 (2013) 7194-7238.

[42] H.M.A. Barbosa, M.M.R. de Melo, M.A. Coimbra, C.P. Passos, C.M. Silva, Optimization of the supercritical fluid coextraction of oil and diterpenes from spent coffee grounds using experimental design and response surface methodology, J. Supercrit. Fluids. 85 (2014) 165-172.

[43] M. Solana, I. Boschiero, S. Dall'Acqua, A. Bertucco, Extraction of bioactive enriched fractions from Eruca sativa leaves by supercritical $\mathrm{CO}_{2}$ technology using different co-solvents, J. Supercrit. Fluids. 94 (2014) 245-251.

[44] G. Brunner, Supercritical gases as solvents: phase equilibria, in: Springer (Ed.), Gas Extr. - An Introd. to Fundam. Supercrit. Fluids Appl. to Sep. Process, Steinkopff-Verlag, New York, 1994: pp. 59-146.

[45] S.P. Jesus, M.N. Calheiros, H. Hense, M.A.A. Meireles, A simplified model to describe the kinetic behavior of supercritical fluid extraction from a rice bran oil byproduct, Food and Publuc Health. 3 (2013) 215-222.

[46] N. Mezzomo, J. Martínez, S.R.S. Ferreira, Supercritical fluid extraction of peach (Prunus persica) 
almond oil: Kinetics, mathematical modeling and scale-up, J. Supercrit. Fluids. 51 (2009) 10-16.

[47] M. Minozzo, A. Popiolski, V.D. Prá, H. Treichel, R.L. Cansian, J.V. Oliveira, A. J. Mossi,M. A. Mazutti, Modeling of the overal kinetic extraction from Maytenus aquifolia using compressed $\mathrm{CO}_{2}$, Braz. J. Chem. Eng. 29 (2012) 835-843.

[48] M.C. Mesomo, M.L. Corazza, P.M. Ndiaye, O.R. Dalla Santa, L. Cardozo, A.D.P. Scheer, Supercritical $\mathrm{CO}_{2}$ extracts and essential oil of ginger (Zingiber officinale R.): Chemical composition and antibacterial activity, J. Supercrit. Fluids. 80 (2013) 44-49.

[49] C. Da Porto, D. Decorti, A. Natolino, Water and ethanol as co-solvent in supercritical fluid extraction of proanthocyanidins from grape marc: A comparison and a proposal, J. Supercrit. Fluids. 87 (2014) 1-8.

[50] H.K. Biesalski, L.O. Dragsted, I. Elmadfa, R. Grossklaus, M. Müller, D. Schrenk, P.Walter, P. Weber, Bioactive compounds: Definition and assessment of activity, Nutrition. 25 (2009) 12021205.

[51] M.R. Kviecinski, P. Benelli, K.B. Felipe, J.F.G. Correia, C.T. Pich, S.R.S. Ferreira, R. C. Pedrosa, SFE from Bidens pilosa Linné to obtain extracts rich in cytotoxic polyacetylenes with antitumor activity, J. Supercrit. Fluids. 56 (2011) 243-248.

[52] L.B. Roseiro, L.C. Duarte, D.L. Oliveira, R. Roque, M.G. Bernardo-Gil, A.I. Martins, C. Sepúlveda, J. Almeida, M. Meireles, F. M. Gírio, A. P. Rauter, Supercritical, ultrasound and conventional extracts from carob (Ceratonia siliqua L.) biomass: Effect on the phenolic profile and antiproliferative activity, Ind. Crops Prod. 47 (2013) 132-138.

[53] P. Benelli, C.A.S. Riehl, A. Smânia Jr., E.F.A. Smânia, S.R.S. Ferreira, Bioactive extracts of orange (Citrus sinensis L. Osbeck) pomace obtained by SFE and low pressure techniques: Mathematical modeling and extract composition, J. Supercrit. Fluids. 55 (2010) 132-141.

[54] P. Costa, C. Grosso, S. Gonçalves, P.B. Andrade, P. Valentão, M. Gabriela Bernardo-Gil, A. Romano, Supercritical fluid extraction and hydrodistillation for the recovery of bioactive compounds from Lavandula viridis L'Hér, Food Chem. 135 (2012) 112-121.

[55] M. Bimakr, R.A. Rahman, F.S. Taip, A. Ganjloo, L.M. Salleh, J. Selamat, A. Hamid, I.S.M. Zaidul, Comparison of different extraction methods for the extraction of major bioactive flavonoid compounds from spearmint (Mentha spicata L.) leaves, Food Bioprod. Process. 89 (2011) 67-72.

[56] K. Ghafoor, J. Park, Y.-H. Choi, Optimization of supercritical fluid extraction of bioactive compounds from grape (Vitis labrusca B.) peel by using response surface methodology, Innov. Food Sci. Emerg. Technol. 11 (2010) 485-490.

[57] R.P. Feliciano, J.J. Meudt, D. Shanmuganayagam, B.T. Metzger, C.G. Krueger, J.D. Reed, Supercritical Fluid Extraction (SFE) of Cranberries Does Not Extract Oligomeric Proanthocyanidins (PAC) but Does Alter the Chromatography and Bioactivity of PAC Fractions Extracted from SFE Residues, J. Agric. Food Chem. (2014).

[58] L. Wang, B. Yang, B. Yan, X. Yao, Supercritical fluid extraction of astaxanthin from Haematococcus pluvialis and its antioxidant potential in sunflower oil, Innov. Food Sci. Emerg. Technol. 13 (2012) 120-127.

[59] B.-C. Liau, C.-T. Shen, F.-P. Liang, S.-E. Hong, S.-L. Hsu, T.-T. Jong, C.-M. J. Chang, Supercritical fluids extraction and anti-solvent purification of carotenoids from microalgae and associated bioactivity, J. Supercrit. Fluids. 55 (2010) 169-175. 
[60] J.L. Pasquel Reátegui, A.P.D.F. Machado, G.F. Barbero, C.A. Rezende, J. Martínez, Extraction of antioxidant compounds from blackberry (Rubus sp.) bagasse using supercritical $\mathrm{CO}_{2}$ assisted by ultrasound, J. Supercrit. Fluids. 94 (2014) 223-233.

[61] R.B. de L. Scodro, S.C. Espelho, C.T. Agostinho Pires, V.A. dos S. Garcia, L. Cardozo-Filho, L.E.R. Cortez, E.J. Pilau, K.R.C. Ferracioli, V.L.D. Siqueira, R.F. Cardoso, D.A.G. Cortez, A new benzoic acid derivative from Piper diospyrifolium and its anti-Mycobacterium tuberculosis activity, Phytochem. Lett. 11 (2014) 18-23.

[62] F.A. Espinosa-Pardo, J. Martinez, H.A. Martinez-Correa, Extraction of bioactive compounds from peach palm pulp (Bactris gasipaes) using supercritical $\mathrm{CO}_{2}$, J. Supercrit. Fluids. 93 (2014) 2-6.

[63] R.G. Bitencourt, C.L. Queiroga, G.H.B. Duarte, M.N. Eberlin, L.K. Kohn, C.W. Arns, F. A. Cabral, Sequential extraction of bioactive compounds from Melia azedarach L. in fixed bed extractor using $\mathrm{CO}_{2}$, ethanol and water, J. Supercrit. Fluids. 95 (2014) 355-363.

[64] C. Da Porto, A. Natolino, D. Decorti, Extraction of proanthocyanidins from grape marc by supercritical fluid extraction using $\mathrm{CO}_{2}$ as solvent and ethanol - water mixture as co-solvent, $\mathrm{J}$. Supercrit. Fluids. 87 (2014) 59-64.

[65] P.C. Veggi, J.M. Prado, G.A. Bataglion, M.N. Eberlin, M.A.A. Meireles, Obtaining phenolic compounds from jatoba (Hymenaea courbaril L.) bark by supercritical fluid extraction, J. Supercrit. Fluids. 89 (2014) 68-77.

[66] H. Bagheri, M.Y. Bin Abdul Manap, Z. Solati, Antioxidant activity of Piper nigrum L. essential oil extracted by supercritical $\mathrm{CO}_{2}$ extraction and hydro-distillation, Talanta. 121 (2014) 220-8.

[67] J. Scapinello, J.V. Oliveira, M.L. Ribeiros, O. Tomazelli, L.A. Chiaradia, J. Dal Magro, Effects of supercritical $\mathrm{CO}_{2}$ extracts of Melia azedarach L. on the control of fall armyworm (Spodoptera frugiperda), J. Supercrit. Fluids. 93 (2014) 20-26.

[68] J. Paes, R. Dotta, G.F. Barbero, J. Martínez, Extraction of phenolic compounds and anthocyanins from blueberry (Vaccinium myrtillus L.) residues using supercritical $\mathrm{CO}_{2}$ and pressurized liquids, J. Supercrit. Fluids. 95 (2014) 8-16.

[69] D.N. e Santos, L.L. de Souza, N.J. Ferreira, A.L. de Oliveira, Study of supercritical extraction from Brazilian cherry seeds (Eugenia uniflora L.) with bioactive compounds, Food Bioprod. Process. (2014) 1-10.

[70] D. Chatterjee, P. Bhattacharjee, Supercritical carbon dioxide extraction of antioxidant rich fraction from Phormidium valderianum: Optimization of experimental process parameters, Algal Res. 3 (2014) 49-54.

[71] N. Mezzomo, L. Tenfen, M.S. Farias, M.T. Friedrich, R.C. Pedrosa, S.R.S. Ferreira, Evidence of anti-obesity and mixed hypolipidemic effects of extracts from pink shrimp (Penaeus brasiliensis and Penaeus paulensis) processing residue, J. Supercrit. Fluids. 96 (2015) 252-261.

[72] D. Cör, T. Botić, Ž. Knez, U. Batista, A. Gregori, F. Pohleven, T. Bončina, Two-stage extraction of antitumor, antioxidant and antiacetylcholinesterase compounds from Ganoderma lucidum fruiting body, J. Supercrit. Fluids. 91 (2014) 53-60.

[73] B. Jayashankar, K.P. Mishra, L. Ganju, S.B. Singh, Supercritical extract of Seabuckthorn Leaves (SCE200ET) inhibited endotoxemia by reducing inflammatory cytokines and nitric oxide synthase 2 expression, Int. Immunopharmacol. 20 (2014) 89-94. 
[74] I.C.M. da Silva, W.L. dos Santos, I.C.R. Leal, M.D.G.B. Zoghbi, A.C. Feirhmann, V.F. Cabral, E.N. Macedo, L. Cardozo-Filho, Extraction of essential oil from Cyperus articulatus L. var. articulatus (priprioca) with pressurized $\mathrm{CO}_{2}$, J. Supercrit. Fluids. 88 (2014) 134-141.

[75] M. Bhattacharya, P.P. Srivastav, H.N. Mishra, Optimization of process variables for supercritical fluid extraction of ergothioneine and polyphenols from Pleurotus ostreatus and correlation to freeradical scavenging activity, J. Supercrit. Fluids. 95 (2014) 51-59.

[76] K. Czaikoski, M.C. Mesomo, A. de Paula Scheer, O.R. Dalla Santa, C.L. Queiroga, M.L. Corazza, Kinetics, composition and biological activity of Eupatorium intermedium flower extracts obtained from $\mathrm{scCO}_{2}$ and compressed propane, J. Supercrit. Fluids. 97 (2015) 145-153.

[77] H.-M. Wang, J.-L. Pan, C.-Y. Chen, C.-C. Chiu, M.-H. Yang, H.-W. Chang, J.-S. Chang, Identification of anti-lung cancer extract from Chlorella vulgaris C-C by antioxidant property using supercritical carbon dioxide extraction, Process Biochem. 45 (2010) 1865-1872.

[78] R.G. Bitencourt, C.L. Queiroga, G.H.B. Duarte, M.N. Eberlin, L.K. Kohn, C.W. Arns, F.A. Cabral, Sequential extraction of bioactive compounds from Melia azedarach L. in fixed bed extractor using $\mathrm{CO}_{2}$, ethanol and water, J. Supercrit. Fluids. 95 (2014) 355-363.

[79] P.B. Gomes, V.G. Mata, A.E. Rodrigues, Production of rose geranium oil using supercritical fluid extraction, J. Supercrit. Fluids. 41 (2007) 50-60.

[80] D. Sparks, R. Hernandez, M. Zappi, D. Blackwell, T. Fleming, Extraction of rice brain oil using supercritical carbon dioxide and propane, J. Am. Oil Chem. Soc. 83 (2006) 885-891.

[81] G. L. Zabot, M. N. Moraes, M. Angela A. Meireles, Supercritical Fluid Extraction of Bioactive Compounds from Botanic Matrices: Experimental Data, Process Parameters and Economic Evaluation, Recent Patents Eng. 6 (2012) 182-206.

[82] S. Vaz Jr, Analytical techniques for the chemical analysis of plant biomass and biomass products, Anal. Methods. 6 (2014) 8094-8105.

[83] B. Tang, W. Bi, M. Tian, K.H. Row, Application of ionic liquid for extraction and separation of bioactive compounds from plants, J. Chromatogr. B. 904 (2012) 1-21.

[84] M.G. Freire, A.F.M. Cláudio, J.M.M. Araújo, J.A.P. Coutinho, I.M. Marrucho, J.N. Canongia Lopes, L.P. Rebelo, Aqueous biphasic systems: a boost brought about by using ionic liquids, Chem. Soc. Rev. 41 (2012) 4966-95.

[85] M.G. Freire, A.R.R. Teles, M.A.A. Rocha, B. Schröder, C.M.S.S. Neves, P.J. Carvalho, D.V. Evtuguin, L.M.N.B.F. Santos, J.A.P. Coutinho, Thermophysical Characterization of Ionic Liquids Able To Dissolve Biomass, J. Chem. Eng. Data. 56 (2011) 4813-4822.

[86] S. Keskin, D. Kayrak-Talay, U. Akman, Ö. Hortaçsu, A review of ionic liquids towards supercritical fluid applications, J. Supercrit. Fluids. 43 (2007) 150-180.

[87] R.E. Teixeira, Energy-efficient extraction of fuel and chemical feedstocks from algae, Green Chem. 14 (2012) 419.

[88] M. Herrero, E. Ibáñez, Green processes and sustainability: An overview on the extraction of high added-value products from seaweeds and microalgae, J. Supercrit. Fluids. 96 (2015) 211-216. 
ionic liquid in a novel assembly of carvacrol, J. Supercrit. Fluids. 61 (2012) 191-198.

[90] M.C. Kroon, J. van Spronsen, C.J. Peters, R. a. Sheldon, G.-J. Witkamp, Recovery of pure products from ionic liquids using supercritical carbon dioxide as a co-solvent in extractions or as an anti-solvent in precipitations, Green Chem. 8 (2006) 246.

[91] M.S. Lenucci, M. De Caroli, P.P. Marrese, A. lurlaro, L. Rescio, V. Böhm, G. Dalessandro, G. Piro, Enzyme-aided extraction of lycopene from high-pigment tomato cultivars by supercritical carbon dioxide, Food Chem. 170 (2015) 193-202.

[92] O. Rachmaniah, Y.H. Choi, I. Arruabarrena, B. Vermeulen, J. van Spronsen, R. Verpoorte, G.-J. Witkamp, Environmentally benign supercritical $\mathrm{CO}_{2}$ extraction of galanthamine from floricultural crop waste of Narcissus pseudonarcissus, J. Supercrit. Fluids. 93 (2014) 7-19.

[93] Y.-J. Fu, W. Liu, Y.-G. Zu, X.-G. Shi, Z.-G. Liu, G. Schwarz, T. Efferth, Breaking the spores of the fungus Ganoderma lucidum by supercritical $\mathrm{CO}_{2}$, Food Chem. 112 (2009) 71-76.

[94] C. Koch, J. Reichling, J. Schneele, P. Schnitzler, Inhibitory effect of essential oils against herpes simplex virus type 2, Phytomedicine. 15 (2008) 71-8.

[95] G.-J. Park, Design of Experiments, Anal. Methods Des. Pract. (2007) 309-391.

[96] K.N. Patel, J.K. Patel, M.P. Patel, G.C. Rajput, H.A. Patel, Introduction to hyphenated techniques and their applications in pharmacy, Pharm. Methods. 1 (2010) 2-13. 
Figure Captions

Figure 1 - The three different extraction periods and its extraction curve.

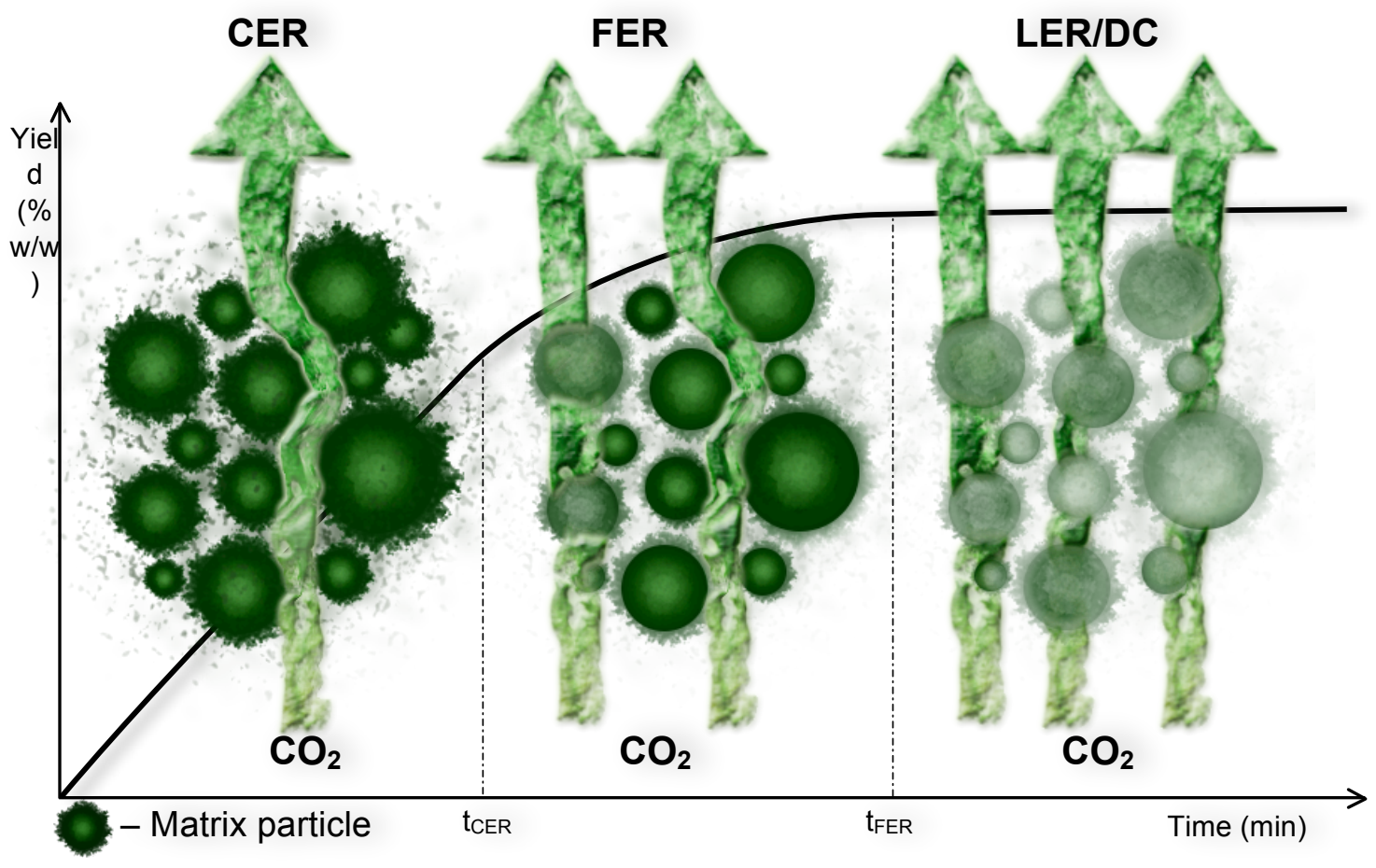

